\title{
L'ANALYSE \\ DE L'INFORMATION \\ Aperçu théorique \\ et application à la loi multinomiale
}

\author{
PAR \\ P. ARBONNIER \\ Directeur de la Station de Biométrie du C.N.R.F.
}





\section{INTRODUCTION}

Les difficultés que suscite l'interprétation des résultats d'un certain nombre d'expériences entreprises par le Laboratoire de Recherches sur le Désherbage ont amené la Station de Biométrie du C.N.R.F. à étudier de plus près les problèmes que pose l'analyse de données recueillies sous forme de notation. L'expérimentation des herbicides en sylviculture a souvent pour objectif la recherche de traitements de contróle - et non de destruction - des espèces adventices forestières: elle exige par conséquent l'appréciation de l'intensité des dommages causés par l'application d'un produit toxique plutôt que la seule observation de la mortalité provoquée. Dans ces conditions, il est impossible d'apprécier l'effet d'un produit autrement que par une notation conventionnelle dont la définition demeure, pour le moment, du ressort de chaque expérimentateur.

L'appréciation par notation n'est pas réservée à cette discipline: de nombreux caractères concernant des arbres ou des plants forestiers ne peuvent être appréciés par une donnée quantitative. Le problème de l'interprétation de résultats d'expériences observés qualitativement intéresse donc l'ensemble de la recherche forestière et, au delà, la recherche agronomique toute entière.

Les difficultés suscitées par l'analyse de données conventionnelles chiffrées sont de plusieurs ordres et contribuent à rendre l'analyse de variance dangereuse ou inopérante. Cette analyse est en effet assortie de conditions dont plusieurs sont, dans ce cas, difficilement satisfaites. Dans tout modèle, même le plus simple tel que:

$$
\mathrm{x}_{1 j}-\mu+\theta_{1}+\varepsilon_{1 s}
$$

l'analyse de variance exige:

1 - que l'erreur $\varepsilon_{1 j}$ soit une variable normale centrée;

2 - que les $\varepsilon_{1 j}$ soient indépendants entre eux;

3 - que la variance des $\varepsilon_{i j}$ soit constante quels que soient $i$ et $j$;

4 - lorsqu'interviennent plusieurs causes contrôlées, on admet en outre l'additivité des effets.

Dans tout système de notations, la variable que constitue la note est une variable discrète qui ne prend que quelques valeurs entières, au plus une dizaine dans la pratique courante. Les hypothèses 1 et 4 sont alors pour le moins difficilement admissibles. L'hypothèse 
3 d'homoscédasticité des erreurs peut ètre contrôlée, par exemple par un test de BARTLETt (encore que ce test soit très sensible à l'absence de normalité). On constate le plus souvent que les variances ne sont pas homogènes.

Il existe encore une ressource; le changement de variables. Ce problème a déjà fait l'objet de nombreuses mises au point [BukGHAUSEN (2), Rrves (9)]. On suggère généralement la transformation arc sin $\sqrt{\mathrm{x}}$ mais bien d'autres ont été proposées depuis les classiques $\log x, \sqrt{x}$ ot $\sqrt{x+1}$ jusquatix scores de Fisher et YATES (5) et des expressions aussi compliquées que:

$$
\mathrm{x}^{\prime}=\text { Probit } \frac{\log (\mathrm{x}+1)}{\log \left(\mathrm{a}_{2}+1-\mathrm{a}_{1}\right)} \quad\left(\mathrm{a}_{2} \text { et } \mathrm{a}_{1} \text { : notes extrêmes }\right)
$$

Le choix d'une variable transiormée est largement facilité par un graphique préalable: DAginele (3), JefFers (6).

Le changement de variable est parfois une solution acceptable, notamment pour les transformations que nous avons qualifiées de classiques, souvent prévues dans les programmes de calculs d'analyse de variance sur calculatrices électroniques, mais l'interprétation des résultats ne s'en trouve pas pour autant facilitée. Enfin, dans certains cas, les chiffres se montrent rebelles à toute transformation: tel est le cas de ceux qui figurent sur le tableau 0.1 qui rassemble les résultats d'une expérimentation de 2.4.5-T pour l'élagage des gourmands de chêne.

Dans une forêt haut-marnaise, une parcelle a été divisée en trois lots et, dans chaque lot, on a choisi 40 chênes couverts de gourmands. Trois traitements, attribués au hasard à chaque lot, ont été appliqués, fin juillet 1962, aux gourmands accessibles aux appareils:

$\mathrm{T} 1$ - une pulvérisation classique à $0,15 \%$ éq. ac. 2.4.5-T,

T2 - une pulvérisation classique à $0,25 \%$ "

T3 - une pulvérisation pneunatique à $1,5 \%$ ” ”

Les résultats ont été observés en 1964 en attribuant à chaque arbre traité, une note conventionnelle de 0 (gourmands totalement détruits) à 6 (gourmands intacts).

\begin{tabular}{|c|c|c|c|c|c|c|c|c|}
\hline Traitement & 0 & 1 & 2 & 3 & 4 & 5 & 6 & \\
\hline T1 & 0 & 0 & 1 & 2 & 20 & 17 & 0 & 40 \\
T2 & 1 & 0 & 0 & 2 & 13 & 23 & 1 & 40 \\
T3 & 2 & 2 & 5 & 6 & 17 & 8 & 0 & 40 \\
\hline Total & 3 & 2 & 6 & 10 & 50 & 48 & 1 & 120 \\
\hline
\end{tabular}

Tableau 0.1 - Résultats : élagage chêne 1962-1964 
Sur cet exemple, aucune transformation, parmi toutes celles qui ont été suggérées par de multiples auteurs, n'a pu homogénéiser les variances. On verra plus loin que l'analyse de l'information permet l'interprétation des résultats de ce tableau.

Lorsque l'interprétation se heurte atinsi à l'impossibilité de mettre en ceuvre toutes les ressources de l'analyse de variance, on doit alors penser à utiliser la méthode fondée sur le test $\chi^{2}$ qui utilise les mémes propriétés d'additivité des estimations et des degrés de liberté. Cette méthode n'est pas tellement simple lorsqu'on veut faire les calculs à la main. Elle nécessite en outre certaines précautions: si les effectifs des cases sont trop faibles, on peut être amené d̀ des regroupements qui, parfois, sont factices et entrainent toujours une perte d'information.

Il existe une méthode heaucoup plus générale d'analyse de données statistiques, qui s'apparente encore du point de vue formel. à l'analyse de variance sans qu'elle implique les mèmes restrictions concernant les lois de distribution des erreurs. Elle dérive de la théorie de linformation et son application ì la loi multinomiale est extrêmement féconde. Récemment signalée par Dufrexoy (4), elle est peu connue des chercheurs qui, dans le domaine agronomique sutant que dans le domaine forestier, y trouveraient souvent la solution du problène difficile que pose linterprétation d'un ensemble d'observations par " notation b.

Ia théoric de l'information et ses applications sont exposées dans des ouvrages sévères susceptibles de dérouter et fle rebuter les chercheurs quii ne sont pas déjà spécialisés en statistique. Plusieur: d'entre eux nous ont demandé d'exposer - sous une forme contensée et qui demeure à leur portée - certaines possibilités offertes à leur discipline par l'analyse de l'information. Tel est le but de cet article qui réstulte de l'analyse de différentes sources mais qui se réfère le plus souvent à l'ouvrage très complet, mais touffu, que Kelzвack (8) a consacré à la théorie de l'information et à ses applications en statictique. II s'inscrit dans le cadre de la mission d'éducation statistique permanente qui constitue une des tâches essentielles de toute station de Biométrie et conditionne son efficacité.

Une première partie est consacrée à un aperçu théorique sur l'information: définition, propriétés, estimation de l'information minima de discrimination. C'est une entreprise difficile que certains jugeront abusive et d'autres — du moins nous l'espérons trop sommaire et schématique. Cependant, il était imprudent de développer les applications de l'analyse de l'information au cas des distributions multinomiales en les présentant conme de simples " recettes de cuisine $n$ : les statisticiens connaissent bien les erreurs l'interprétation qui peuvent résulter d'un usige abusif de telles recettes. Nous offrons donc à l'utilisateur en puissance la possibilité de s'assurer qu'il emploie à bon escient les méthodes d'analyse 
proposées; peut-être aura-t-il ensuite la curiosité d'approfondir ces problèmes et d'en connaitre les développements dans d'autres domaines, tels que l'analyse multivariable $\left({ }^{*}\right)$.

Une seconde partie expose les applications pratiques de l'information en se limitant au cas particulier de la distribution multinomiale qui englobe les tables de contingence à double et triple entrée. Les différents tests d'homogénéité d'échantillons d'une loi multinemiale, de mème que les nombreux tests d'indépendance des classifications de tables de contingence sont illustrés par des exemples empruntés aux dossiers de la Station de Biométrie. Ces exemples sont assez nombreux pour que le lecteur, désireux d'appliquer les méthodes exposées, puisse s'assurer qu'il les emploie correctement. Une table de $\mathrm{n} \log \mathrm{n}$ à 4 décimales pour $\mathrm{n} \leqslant 200$ devrait permettre la plupart des calculs courants (**).

(*) Ce sujet est développé par Kullasck (8) qui y consacre plus de 150 pages.

(**) Cette table a été obtenue sur la calculatrice C A E 510 du Centre de Calcul Automatique de l'Université de Nancy. Nous remercions M. le Professeur I.zGkas, ainsi que M. Toxassonf qui a bien voulu rédiger (en ALGOL) le petit programme nécessaire. On peut encore obtenir de la Station de Biométrie du C.N.R.F., la table des valeurs de $n \log n$ ( 4 décimales) pour $200<n \leqslant 1000$. Bien que le programme soit très simple, il peut être également mis à la disposition de tout utilisateur d'une calculatrice équipée d'un compilateur ALGOL. 


\section{DÉFINITION ET PROPRIETTESS DE L'INFORMATION ET DE SON ESTIMATION}

0. - La notion d'information se rattache à la connaissance de l'incertain et, dès lors, est inséparable de celle d'événement aléatoire. Or, l'algèbre des événements est différente de l'algèbre des nombres: les mathématiciens ont dù imaginer de nouveaux concepts pour élargir le champ où s'exerce leur logique: la théorie des probabilités a largemenu participé mais doit aussi beaucoup aux progrès d'une théorie plus générale encore, la théorie de la mesure, qui constitue un domaine avancé des mathématiques modernes.

Un exposé, même très succinct, de la théorie de l'information ne saturait donc, sans sacrifier à la généralité et à la rigueur du raisonnement, se préserver d'une certaine abstraction. Pourtant, le point de départ est intuitif et, historiquement, les notions d'entropic et d'information sont nées de l'étude de problèmes pratiques, notamment ceux que pose la transmission par fil des informations (HAkTLEY, SHANNoN) $\left(^{\circ}\right)$. La notion d'information s'impose à l'observateur de tout phénomène aléatoire.

Un exemple l'illustrera. Une urne renferme quatre boules et l'on sait que celles-ci peuvent être, soit toutes blanches, soit deux blanches et deux noires; ce sont là deux hypothèses $H_{1}$ et $H_{2}$ entre lesquelles on s'efforce de discriminer la bonne. On tire une première boule de l'urne, ce qui constitue une épreuve dont le résultat (aléatoire) est la couleur de la boule, Si la boule est noire, on peut immédiatement écarter $\mathrm{H}_{1}$ ( 4 blanches) au profit de $\mathrm{H}_{2}$ ( 2 blanches +2 noires). Si la boule est blanche on n'en tire aucune conclusion immédiate. La première boule étant replacée dans l'urne, on effectue, après brassage, un second tirage. Dans l'hypothèse où la boule

(*) Sans oublier la thermodynamique où l'entropie est une fonction d'état. 
est encore blanche (conclusion différée), on peut. par application du postulat et du théorène de Bayss, conclure que la probabilité de $\mathrm{H}_{1}$ est augmentée $\left(^{*}\right)$. Ainsi le résultat de chaque épreuve. quel quil soit, nous a apporté une information qui a contribué chaque fois à réduire l'incertitude initiale: l'information dépend du résultat de l'éprenve et de la nature des hypothèses.

\section{Définition de l'information - Divergence \\ 1. 1 - L'information.}

Soit deux mesures de probabilités définies dans un esnace de probabilité $\Omega$ par:

$$
\begin{array}{cl}
\mu_{1}(\mathrm{E})=\int_{\mathrm{E}} \mathrm{f}_{1}(\mathrm{x}) \mathrm{d} \lambda(\mathrm{x}) & \mathrm{i}=1,2 \\
\text { pour tout } \mathrm{E} \in \mathrm{dl}(\mathrm{*}) \text { et } \lambda(\mathrm{E}) \neq 0 & \\
\text { ce qui séerit aussi } \frac{\mathrm{d} \mu_{1}}{\mathrm{~d} \lambda}-\mathrm{f}_{1}(\mathrm{x}) & \text { (h) }\left(^{*}\right)
\end{array}
$$

$f_{1}(x)$ est la densité de prubabilité généralisée $0<f_{1}(x)<\infty$

Si $\mathrm{H}_{1}$ désigne l'hypothèse stuivant laquelle la variable aléatoire $\mathrm{X}$ appartient à la population dont la mesure de probabilité est $\mu_{i}$, il lui correspond tune certaine probabilité à priori $\mathrm{P}\left(\mathrm{H}_{\mathrm{i}}\right)$. Lorsque $\mathrm{X}$ prend une valeur déterminée $x$, on peut encore définir une probabilité à postériori, que l'on écrit $\mathrm{P}\left(\mathrm{H}_{1} / \mathrm{x}\right)$. ot probahilité condition-

(*) Cf. Kexdalt. (7), vol, 2, page 199 .

${ }^{(* *)} \mu_{i}$ est une mesure de prohabilité au sens général. Elle est définie sur un espace de probabilit $(\Omega, a)$ éest-a-dire dans un espace $\Omega$ (ensemble des événements élémentaires of muni d'une $\sigma$-algèbre (ou corps de Borel) el, On suppose en outre que $\mu_{1}$ et $\mu_{2}$, sont ahsolument continues lune par rapport it lautre, ce qui s'écrit:

$$
\mu_{1} \ll \mu_{2} \quad \mu_{2} \ll \mu_{1} \quad \mu_{1}: \mu_{2}
$$

et gu'il existe une mesure $\lambda$ telle que:

$$
\lambda \equiv \mu_{1} \quad \lambda \equiv \mu_{2}
$$

$\mu_{1}$ et $\mu_{\text {, }}$ appartiennent à un ensemble dominé par $\lambda$ ).

La densité de probabilité généralisée. $f_{1}(x), i=1,2$ est définie en vertu du theorème de Radon-Nikodym; woir par exemple Tortrat (11), chap. V.

(*) (a) se lit \& modulo $\lambda$ \& u signifie que la relation qui précède est vraie i. lexclusion des ensembles $\mathrm{i}$ appartenant à $\mathrm{ol}$ (Eech) et de mesure $\lambda$ nulle. 
nelle de $\mathrm{H}_{\mathrm{i}}, \mathrm{X}-\mathrm{x}$ étant donné. L'application du théorème de BAYEs permet d'écrire

$$
\mathrm{i}=1,2
$$

$$
P(H 1 / x)=\frac{P(H 1) f 1(x)}{P\left(H_{1}\right) f_{1}(x)+P\left(H_{2}\right) f_{2}(x)}
$$

En donnant successivement à $\mathrm{i}$ les valeurs 1 et 2 dans (1, 2) et en divisant membre à membre, il vient:

$$
\frac{P\left(H_{1} / x\right)}{P\left(H_{2} / x\right)}=\frac{P\left(H_{1}\right) f_{1}(x)}{P\left(H_{2}\right) f_{2}(x)}
$$

ou encore, quelle que soit la base des logarithmes,

$$
\log \frac{f_{1}(x)}{f_{2}(x)}=\log \frac{P\left(H_{1} / x\right)}{P\left(H_{2} / x\right)}-\log \frac{P\left(H_{1}\right)}{P\left(H_{2}\right)}
$$

Au second membre apparait la différence de deux termes liés aux chances favorables à $\mathrm{H}_{1}$ : le premier terme (apiès l'épreuve) implique que l'on a observé une valeur $\mathrm{X}=\mathrm{x}$; le second terme (avant l'épreuve) l'ignore.

Le premier membre $\log \left[\mathrm{f}_{1}(\mathrm{x}) / \mathrm{f}_{\mathrm{g}}(\mathrm{x})\right]$ définit alors l'information apportée par $X=x$ en faveur de lhypothèse $H_{t}$. Il peut être positif ou négatif; c'est également le logarithme du rapport de vraisemblance.

L'information moyenne, relativement à $\mu_{1}$ est, pour :

$$
\mathrm{x}: \mathrm{x} \in \mathrm{E} \in \mathrm{Cl}
$$

$$
I(1: 2 ; E)=\frac{1}{\mu_{1}(E)} \int E \log \frac{f_{1}(x)}{f_{2}(x)} d \mu_{1}(x)
$$

$$
\begin{aligned}
(1,4) & =\frac{1}{\mu_{1}(E)} \int_{E} f_{1}(x) \log \frac{f_{1}(x)}{f_{2}(x)} d \lambda(x) & & \mu_{1}(E)>0 \\
& =0 & & \mu_{1}(E)=0
\end{aligned}
$$


Si E s'étend à lespace de probabilité tout entier $\mathbf{\Omega}$ on écrit:

$$
\begin{aligned}
& I(1: 2)=\int \log \frac{f_{1}(x)}{f_{2}(x)} d \mu_{1}(x)=\int f_{1}(x) \log \frac{f_{1}(x)}{f_{2}(x)} d \lambda(x) \\
& =\int \log \frac{P\left(H_{1} / x\right)}{P\left(H_{2} / x\right)} d \mu_{1}(x)-\log \frac{P\left(H_{1}\right)}{P\left(H_{2}\right)}
\end{aligned}
$$

En effet $\mu_{1}(\Omega)-1$ par définition et $d \mu_{1}(x)=f_{1}(x)$ d $\lambda(x)$

I(1 : 2) est linformation moyenne de discrimination en faveur de $H_{1}$ (contre $\mathrm{H}_{2}$ ) ou, plus succinctement, l'information de $\mu_{1}$ par rapport à $\mu_{2}$.

On peut définir de façon analogue l'information de $\mu_{2}$ par rapport à $\mu_{1}$.

(1. 6)

$$
I(2 ; 1)=\int f_{2}(x) \log \frac{f_{2}(x)}{f_{1}(x)} d \lambda(x)
$$

1. 2 - La divergence.

On peut aussi définir une expression symétrique par rapport à $\mathrm{H}_{1}$ et $\mathrm{H}_{2}$, la divergence $J(1,2)$ :

$$
\begin{gathered}
J(1,2)=I(1 ; 2)+I(2: 1) \\
=\int\left(f_{1}(x)-f_{2}(x)\right) \log \frac{f_{1}(x)}{f_{2}(x)} d \lambda(x) \\
=\int \log \frac{p\left(H_{1} / x\right)}{p\left(H_{2} / x\right)} d \mu_{1}(x)-\int \log \frac{p\left(H_{2} / x\right)}{p\left(H_{1} / x\right)} d u_{2}(x)
\end{gathered}
$$

expression d'où ont disparu les probabilités à priori $\mathrm{p}\left(\mathrm{H}_{1}\right)$ et $\mathrm{p}\left(\mathrm{H}_{2}\right)$.

$J(1,2)$ possède certaines propriétés axiomatiques des distances $\left({ }^{*}\right)$. Elle mesure la difficulté de discriminer $\mathrm{H}_{1}$ de $\mathrm{H}_{2}$. L'infor-

(*) A l'exception de celle dite * inégalité triangulaire *:

$$
\overline{\mathrm{AB}}+\overline{\mathrm{BC}}>\overline{\mathrm{AC}}
$$


mation I $(1: 2)$ peut, de la même façon, être considérée comme une mesure de la divergence orientée entre $\mathrm{H}_{1}$ et $\mathrm{H}_{2}$.

1. 3 - Exemple - Distribution multinomiale.

Chaque population est constituée d'éléments appartenant à c classes auxquelles les hypothèses $H_{1}$ attachent les probabilités $p_{11}$

$$
i=1,2 \quad j=1,2 \ldots c .
$$

Lapplication de la formule (1, 5) at cas d'une variable discrète donne:

$$
\begin{array}{r}
I(1 ; 2)=\sum_{j=1}^{c} p_{1 j} \log \frac{p_{1 j}}{p_{2 j}}=p_{11} \log \frac{p_{11}}{p_{21}}+p_{12} \log \frac{p_{12}}{p_{22}} \\
\ldots+p_{1 c} \log \frac{p_{1 c}}{p_{2 c}}
\end{array}
$$

de même

$$
I(2: 1)=\sum_{J} p_{2 J} \log \frac{p_{2 J}}{p_{1 j}}
$$

et

$$
J(1,2)=\sum_{J}\left(p_{1 J}-p_{2 J}\right) \log \frac{p_{1 J}}{p_{2 j}}
$$

\section{2 - Propriétés de l'information}

\section{1 - Additivité.}

L'information est additive. $\mathrm{Si}$ deux événements aléatoires $\mathrm{X}$ et $\mathrm{Y}$ sont indépendants, alors:

$$
\mathrm{I}(1: 2 ; \mathrm{X}, \mathrm{Y})-\mathrm{I}(1: 2 ; \mathrm{X})+\mathrm{I}(1: 2 ; \mathrm{Y})
$$

En particulier un échantillon de $\mathrm{n}$ observations indépendantes fournit $\mathrm{n}$ fois l'information apportée par une seule observation, ce qui satisfait à une exigence intuitive. L'additivité est du reste une condition imposée à priori lorsqu'on définit de façon axiomatique l'indétermination d'une expérience, par exemple l'entropie [cf. Yaglom et Yaglom (13), p. 34]. 
Si les variables ne sont pas indépendantes, l'additivité demeure mais en fonction de probabilités conditionnelles:

$$
\mathrm{I}(1: 2 ; \mathrm{X}, \mathrm{Y})=\mathrm{I}(1: 2 ; \mathrm{X})+\mathrm{I}(1: 2 ; \mathrm{Y} / \mathrm{X})
$$

\section{2. $2-$ Converité.}

L'information est toujours positive sauf lorsque $f_{1}(x)=f_{2}(x)$ auquel cas elle est nulle.

$$
\mathrm{I}(1: 2) \geqslant 0 \quad \mathrm{I}(1: 2)=0=>\mathrm{f}_{1}(\mathrm{x})=\mathrm{f}_{2}(\mathrm{x})
$$

Si les deux densités sont les mẻmes, aucune discrimination n'est possible par l'information.

On montre encore que si lon divise un ensemble $\mathrm{E}$ en un certain nombre de sous-ensembles disjoints $\mathrm{E}_{1}$ tels que $\mathrm{U}_{1} \mathrm{E}_{1}=\mathrm{E}$ la somme des informations relatives à chaque sous-ensemble $E_{1}$ est inférieure à l'information globale sur $\mathrm{E}$ : autrement dit le groupement des données diminue l'information; on dit encore que l'information est une fonction convexe (comme l'est aussi la fonction $\mathrm{t} \log \mathrm{t})$.

\section{3 - Transformations.}

L'information n'est généralement pas conservée (invariante) lorsqu'on opère une transformation des données (choix d'une statistique); sa valeur diminue sauf si la statistique choisie $\mathrm{T}(\mathrm{x})$ satisfait la relation:

$$
\frac{f_{1}(x)}{f_{2}(x)}=\frac{g_{1}(T(x))}{g_{2}(T(x))}
$$

$g_{1}, g_{2}$ densité de probabilité

de la variable transformée

La statistique $y-\mathrm{T}(\mathrm{x})$ est alors dite " stuffisante $n$.

En résumé, linformation est additive mais le regroupement, la condensation ou la transformation des données entrainent le plus sowvent une perte d'information. Dans l'hypothèse d'une statistique suffisante, l'information est conservée mais jamais accrue.

\section{3 - Information minima de discrimination}

\section{1 -Définition.}

La valeur de l'information constitue une mesure de la divergence " orientée $n$ qui sépare l'hypothèse $\mathrm{H}_{1}$ de l'hypothèse $\mathrm{H}_{2}$; à ce 
titre, dle peut permettre de discerner, dans un ensemble (dominé) de mesures de probabilité $\mu_{1}$ quelle est celle qui est la plus proche de $\mu_{2}$. en d'autres termes, la plus ressemblante: c'est celle qui fournira la plus petite valeur de $\mathrm{T}(1: 2)$.

On a vu que $\mathrm{I}(1: 2) \geqslant 0$ : légalité correspond à $\mathrm{f}_{1}(\mathrm{x})-\mathrm{f}_{\mathrm{g}}(\mathrm{x})$ quii, dans notre cas, est sans intérêt.

Nous rechercherons donc un minimum lić à une relation imposéc quii peut être la condition qu'une statistique mesurable définie par $\mathrm{Y}=\mathrm{T}(\mathrm{x})$ ait pour espérance mathématique une valeur donnée $\theta$ pour $\mu_{1}$.

Cette condition sexprime sots forme analytique par:

$$
\int \mathrm{T}(\mathbf{x}) \mathrm{d} \mu_{1}(\mathbf{x})=\int \mathrm{T}(\mathbf{x}) \mathrm{f}_{1}(\mathbf{x}) \mathrm{d} \lambda(\mathbf{x})=\theta \quad \mathrm{E}_{1}(\mathrm{~T}(\mathbf{x}))=\theta
$$

$\theta$ étant un paramètre des poptulations qui, comme $\mathrm{x}$, peut être multidimensionnel (et qu'on se propose par la suite d'estimer sur un ou plusieurs échantillons).

En associant $f_{2}(x)$ à l'ensemble des poptulations de l'hypothèse nulle et $f_{1}(x)$ à celles de l'hypothèse alternative - dont l'échantillon constitue un membre possible - en minimisant la valeur de l'information, on sélectionnera celle des populations de densité $\mathrm{f}_{\mathrm{z}}(\mathrm{x})$ qui est la plus proche. c'est-ì-dire la plus ressemblante à l'échantillon. Si cette ressemblance est trop faible, on rejettera l'hypothèse nulle; en terme d'information, le rejet de l'hypothèse nulle interviendra si la valeur du minimum ou o information minima de discriminatiosn " lépasse le seuil de signification que l'on s'est fixé.

On démontre le théorème suivant - Kullasck (8), p. 38:

Théorìme: Si $\mathrm{f}_{1}(\mathrm{x})$ et une $\mathrm{f}_{2}(\mathrm{x})$ donnée sont les densités de probabilités généralisées d'un ensemble dominé de mesures de prohabilités, et si $\mathrm{Y}=\mathrm{T}(\mathrm{x})$ est une statistique mesurable telle que:

$$
0=\int T(x) f_{1}(x) d \lambda(x)
$$

existe

et

$$
M_{2}(\tau)=\int f_{2}(x) e^{\tau T(x)} d \cdot \lambda(x)
$$

existe sur un certain intervalle de $\tau$, alors: 
(3. 2)

$I(1: 2) \geqslant \theta \tau-\log M_{2}(\tau)=I(\bullet: 2$ $\theta=\frac{d}{d \tau} \log M_{2}(\tau)$

avec égalité si :

(3. 3)

$$
f_{1}(x)=f^{*}(x)=e^{\tau T(x)} \cdot f_{2}(x) / M_{2}(\tau)
$$

Lorsque - varie sur son intervalle $f^{*}(x)$ engendre une famille exponentielle de distrihution (*).

3. 2 - Application a la loi multinomiale.

Pour une distribution multinomiale où l'effectif total $\mathrm{N}$ est réparti en $c$ classes, d'effectif, respectifs $x_{1}, x_{2} \ldots x_{e}$

$$
\mathrm{x}_{1}+\mathrm{x}_{2}+\ldots \mathrm{x}_{\mathrm{e}}=\mathrm{N}
$$

et auxquelles sont attachées les probabilités $p_{1}, p_{2} \ldots p_{e}$

$$
p_{1}+p_{2}+\ldots p_{r}=1
$$

lit foi de distribution est:

$$
p(x)=p\left(x_{1}, x_{2}, \ldots x_{c}\right)=\frac{N 1}{x_{1}\left|x_{2}\right| \ldots x_{c}} p_{1}{ }^{x_{1}} p_{2}{ }^{x_{2}} \ldots p_{c}{ }^{x_{c}}
$$

la variable $\mathrm{x}$ est multidimensionnelle (c dimensions) et discrète.

La distribution conjuguée $\mathrm{p}^{*}(\mathrm{x})$ donnant l'information minima de discrimination par rapport à la distribution de densité $p(x)$ et satisfaisant à la condition:

$$
\mathrm{E}^{*}(\mathrm{x})=\theta \text {, qui implique } \mathrm{T}(\mathrm{x})-\mathrm{x} \text { dans (3. 1) }
$$

(*) Ce théorème introduit la fonction exponentielle $\mathrm{e}^{\tau \mathrm{T}(\mathrm{x})}-$ que nous écrivons aussi exp $[\div \mathrm{T}(\mathrm{x})]$, notamment lorsque $\mathrm{x}$ et $:$ sont des variables à plusieurs dimensions - dont l'inverse définit la fonction logarithme. Il en résulte que désormais les logarithmes utilisés sont népériens (base e). 
soit $\quad E^{*}\left(x_{1}\right)=\theta_{1} \quad i-1,2 \ldots c-\theta$ étant ici un paramètre défini dans l'espace à c-dimensions - est:

$$
\begin{aligned}
& p *(x)=p(x) \frac{\exp \left(\tau_{1} x_{1}+\tau_{2} x_{2}+\ldots+\tau_{c} x_{c}\right)}{\left(p_{1} e^{\tau_{1}}+p_{2} e^{\tau_{2}}+\ldots p_{c}{ }^{{ }^{\tau} c}\right)^{N}} \\
& =\frac{N !}{x_{1} ! x_{2} ! \cdots x_{c} !}\left(p_{1}{ }^{*}\right)^{x_{1}}\left(p_{2}{ }^{*}\right)^{x_{2}} \ldots\left(p_{c}{ }^{*}\right)^{x_{c}}
\end{aligned}
$$

où

(3. 6)

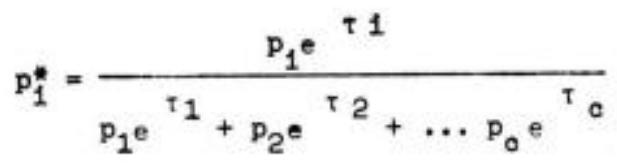

les $\tau$ । étant des paramètres réels définis par

(3. 7)

$$
\begin{gathered}
\theta_{i}=\frac{\partial}{\partial \tau_{i}} \log \left(p_{1} e^{\tau} 1+p_{2} e^{\tau}+\ldots \cdots p_{c} e^{\tau} c^{N} N\right. \\
\text { puisque } \theta_{i}=\frac{\partial}{\partial \tau_{i}} \log M_{2}(\tau)
\end{gathered}
$$

On remarque que la distribution d'information minima est encore multinomiale.

(*) On démontre facilement que:

$$
M_{2}(\tau)=\left(p_{1} e^{\tau 1}+p_{2} e^{\tau 2}+\ldots+p_{c} e^{\tau c}\right)^{N}
$$

car

$$
M_{2}(\tau)=x_{1}+x_{2}^{[} \ldots x_{0}=p(x) e^{\tau x}
$$

dans le cas d'une variable discrète à cadimensions. En remplaçant $p(x)$ par sa valeur de $(3,4)$ et e $\tau^{x}$ par exp $\left(\tau_{1}-x_{1}+\ldots, x_{f}\right)$ on obtient le développement de la parenthèse ci-dessus. 
D'après (3. 7)

$$
0_{1}=N p_{1} e^{\tau} 1 /\left(p_{1} e^{\tau_{1}}+p_{e_{2}} e^{\tau_{2}}+\ldots p_{c} e^{T_{c}}\right)=N p_{1}^{*}
$$

d'ou l'on tire $\tau_{1}$

$$
\begin{aligned}
& \tau_{1}=\log \left(\theta_{1} / \mathrm{Np}_{1}\right)+\log k \text { aveo } k=p_{1} e^{\tau^{1}}+p_{2} e^{\tau_{2}}+\ldots+p_{c} e^{\tau_{c}}>0 \\
& \left.I *: 2 ; O_{N}\right)=\sum_{x_{1}}+x_{2}+\ldots=N
\end{aligned}
$$

$=\tau_{1} o_{1}+\tau_{2}, \ldots+\ldots+\tau_{2} e_{c}-W \log k$

$I\left(*, 2 ; O_{N}\right)=\theta_{1} \log \frac{\theta_{1}}{\mathrm{~Np}_{1}}+\theta_{2} \log \frac{\theta_{2}}{\mathrm{~Np}_{2}}+\ldots \ldots+\theta_{c} \log \frac{\theta_{\mathrm{c}}}{\mathrm{Np}_{\mathrm{c}}}$

Puisque $\mathrm{K}$ est arbitraire, on peut prendre $\mathrm{K}=1$ et alors

$$
\tau-\log \left(\theta_{1} / \mathrm{N}_{p_{1}}\right)
$$

\section{4 - Statistiques d'information}

4. 1 - listimation de Pinformation.

Nous avons jusqu’à présent considéré $\theta$ comme un paramètre connu de la population et nous avons défini à partir de ce paramètre la distribution conjuguée $f^{*}(x)$ qui fournit l'information minima de discrimination: $1\left({ }^{\circ} ; 2\right)$ donnée par la formule (3. 2).

Sur un échantillon de $\mathrm{n}$ observations indépendantes extraites d'une population de densité de probabilité $\mathrm{f}_{2}(\mathrm{x})$, nous utiliserons la valeur observée de la statistique $\mathrm{T}(\mathrm{x})$ comme une estimation $\hat{\theta}(\mathrm{x})$

(1) $\left.\mathrm{I}^{*}: 2 ; \mathrm{Ox}\right)$ désigne l'information fournie par l'échantillon ON d'effectif $\mathrm{N}=\mathrm{x}_{1}+\mathrm{x}_{2}+\ldots .+\mathrm{x}_{n}$ 
de $\theta$. A la valeur $\hat{\theta}$ de $\theta$ correspond une estimation $\hat{\tau}$ de $\tau$ implicite dans:

$$
T(x)=\hat{\theta}(x)=\left(\frac{c}{d \tau} \log M_{2}(\tau)\right)_{T=\hat{T}} \hat{\tau}(x)-\tau(\theta(x))
$$

Alors l'estimation de I est 1 :

$$
\hat{I}\left(*: 2 ; o_{n}\right)=\hat{\theta}(x) \cdot \hat{\tau}(x)-\log M_{2}(\hat{\tau}(x))
$$

S'il existe plusieurs estimateurs sans biais $\hat{\theta}(x)$ de $\theta$, nous choisirons celui qui domne la plus grande valeur de $I\left({ }^{*}: 2\right)$ assurant ainsi le meilleur pouvoir séparateur pour la discrimination. De touttes façons $I(*: 2: 0)$ dans la formule $(4,2)$ est l'information minima de discrimination entre une population de densité de probabilité de la forme $\mathrm{f}^{*}(\mathrm{x})$, pour laquelle la valeur du paramètre $\theta$ est la mème que celle observée sur l'échantillon et la population dont la densité de probabilité est $\mathrm{f}_{2}(\mathrm{x})$. Plus la valeur de l'information est grande, moins l'échantillon ressemble à la poptulation $f_{2}(x)$.

\section{Exemple: distribution multinomiale.}

I.'hypothèse $\mathrm{H}_{2}$ suppose que l'échantillon d'effectif $\mathrm{N}$ appartient i) la clistribution multinomiale caractérisée par les probabilités pi

$$
\mathrm{i}=1,2 \ldots \mathrm{c} \quad \boldsymbol{\Sigma}_{1} \mathrm{p}_{1}=1
$$

on prendra pour meilleur estimateur de $\theta_{1}=\mathrm{Np}_{1}{ }^{*}$

$$
\hat{\theta}_{i}-x_{1} \text { effectif constaté clans la classe } i \text {. }
$$

Alors, en vertu de $(3,9)$ et $(3,8)$

$$
\hat{\tau}_{1}-\log \frac{\mathrm{x}_{1}}{\mathrm{~Np}_{\mathrm{p}}}
$$

$$
\hat{I}\left(*: 2 ; 0_{N}\right)=x_{1} \log \frac{x_{1}}{N_{1}}+x_{2} \log \frac{x_{2}}{N_{2}}+\ldots x_{c} \log \frac{x_{c}}{N p_{c}}
$$


formule que l'on peut rapprocher te (1.8) où l'on ferait $p_{1}-p_{21}$ et $\mathrm{x}_{1}$

$\frac{x_{1}}{\mathrm{~N}}=\mathrm{p}_{1 \mathrm{i}}$, avec naturellement $\mathrm{I}\left(1: 2 ;()_{\mathrm{N}}\right)=\mathrm{N} \mathrm{I}(1 ; 2)$. $\mathrm{N}$

Ia formule (4, 4) sera largement utilisée dans les applications pratiques.

\section{2 - Classement d'hypothèses.}

II peut se faire qu'un échantillon ON puisse ètre observé sous plusieurs hypothèses $H_{1}$ exhaustivez et mutuellement exclusives auxquelles sont attachées les prohabilités à priori $\mathrm{P}\left(\mathrm{H}_{1}\right)$

$$
\mathrm{P}\left(\mathrm{H}_{\mathrm{i}}\right)>0 \quad \Sigma_{\mathrm{i}} \mathrm{P}\left(\mathrm{H}_{1}\right)=1 \quad \mathrm{i}=1,2 \ldots \mathrm{r}
$$

On démontre que si les probabilités conditionnelles de $O_{N}$ appartiennent à une famille exponentielle [telle que $\left.f^{*}(x)\right]\left({ }^{*}\right)$, la plus petite valeur $\mathrm{I}\left({ }^{*}: \mathrm{m}\right) 1 \leqslant \mathrm{~m} \leqslant \mathrm{r}$ de l'estimation $\mathrm{I}(* ; \mathrm{i})$ - information minima de discrimination pour $\mathrm{H}_{1}$ - est attachée à la population pour laquelle le rapport de la probabilité a posteriori $\mathrm{P}\left(\mathrm{H}_{1} / \mathrm{ON}\right)$ à la probabilité a priori $\mathrm{P}\left(\mathrm{H}_{4}\right)$ est maximum.

Cétte valeur est une mesure de la divergence orientée entre l'échantillon et le membre de la famille $\mathrm{H}$ (des hypothèses $\mathrm{H}_{1}$ ) dont il se rapproche le plus. On l'écrit alors $\mathrm{I}\left({ }^{*} ; \mathrm{H}\right)$; c'est la statistique d'information minima de discrimination.

\section{3 - Test d'hypothèse.}

I.onsquion désire tester une hypothèse nulle $\mathrm{H}_{2}$ par rapport à une hypothèse alternative $H_{1}$, on pourra le faire en se fixant la valeur $\alpha$ du risque de première espéce (probabilité de rejeter $\mathrm{H}_{2}$ lorsque l'échantillon appartient aux populations de $\mathrm{H}_{2}$ ).

L'hypothèse $\mathrm{H}_{2}$ sera rejetée si

$$
\text { Prob. }\left[\hat{\mathrm{I}}\left(* ; \mathrm{H}_{2}\right)-\mathfrak{I}\left(* ; \mathrm{H}_{1}\right) \geqslant \mathrm{C} / \mathrm{H}_{2}\right] \leqslant \alpha
$$

\section{4 - Propriétés asymptotiques.}

Les propriétés asymptotiques de la statistique d'information minima ont fait l'objet de nombrenses recherches souvent liées à celles des propriétés asymptotiques du logarithme du rapport de vraisemblance et plusieurs théorènes ont établi (Wit.ks, Kupperman) que sous certaines conditions ce rapport suivait une loi de $\chi^{2}$, centré dans l'hypothèse nulle et non centré dans l'hypothèse alternative.

(*) La plupart des lois de distribution classiques: loi normale, loi multinomiale, loi de Poisson, loi de $\gamma^{2}$, etc. satisfont â cette restriction. 
Plus généralement, on démontre $\left(^{*}\right)$ que si l'échantillon appartient à la population de densité de probabilité $\mathrm{f}_{2}(\mathrm{x})$ pour laquelle $\mathrm{I}(*: 2 ; \mathrm{ON})=0$ l'estimation I de I est telle que

$2 \Upsilon$ (* : 2 ; ON) est asymptotiquement distribuée comme $\chi^{2}$ avec $\mathrm{k}$ degrés de liberté, $\mathrm{k}$ étant la dimension du paramètre $\theta$ : par exemple $\mathrm{c}-1$ dans le cas d'une distribution multinomiale à c classes (où $\mathbf{\Sigma} \mathrm{x}_{1}=\mathrm{n}$ constitue une liaison entre les $\mathrm{x}_{1}$ ).

On peut alors déterminer un intervalle de confiance asymptotique 1 - $\alpha$ pour les paramètres de $\mathrm{f}_{2}(\mathrm{x})$ en écrivant

$$
2 \hat{\mathrm{I}}\left(\%: 2: \mathrm{ON}_{\mathrm{N}}\right) \leqslant \chi^{2}(\mathrm{k}, \alpha)
$$

$\chi^{2}(\mathrm{k}, \alpha)$ étant la valeur lue sur la table de $\gamma^{2}$, pour $\mathrm{k}$ degrés de liberté au seuil de probabilité $\alpha$

$$
\text { Prob. }\left[\chi^{2} \geqslant \chi^{2}(K, \alpha)\right]=\alpha
$$

La fonction $\left.2 \mathrm{I}^{*}: 2 ; \mathrm{O}_{\mathrm{N}}\right)$ étant une fonction convexe, on obtient deux limites qui déterminent entre elles l'intervalle de confiance du paramètre estimé de $\mathrm{f}_{2}(\mathrm{x})$.

On démontre également que lorsque l'échantillon n'appartient pas à la poptulation de densité $\mathrm{f}_{2}(\mathrm{x}) 2 \mathrm{I}\left(*: 2 ; \mathrm{ON}_{\mathrm{N}}\right)$ stit asymptotiquement une distribution de $\chi^{2}$ non centré, avec $\mathrm{k}$ degrés de liberté, le paramètre de décentrage étant $2 \mathrm{I}\left({ }^{*}: 2 ; \mathrm{ON}_{\mathrm{N}}\right)$ dont la valeur est nulle dans l'hypothèse nulle.

On démontre enfin que la divergence $J(* ; 2)$ définie comme au paragraphe 1. 2 pour la loi $\mathrm{f}^{*}(\mathrm{x})$ est asymptotiquement égale à $2 \mathrm{I}\left({ }^{2}: 2\right)$ et par conséquent suit asymptotiquement la loi de $\chi^{2}$ dans l'hypothèse nulle et de $\gamma^{2}$ non centré dans Thypothèse alternative.

Cas particulier: loi multinomiale.

Dans le cas particulier de la loi multinomiale, on peut montrer assez facilement l'approximation de $2 \hat{\mathrm{I}}(* ; 2)$ par une loi de $\chi^{2}$. On a pour $\mathrm{a}>0$ b $>0$

$$
\frac{a-b}{a} \leqslant \log a / b \leqslant \frac{a-b}{b}(\log x \leqslant x-1 \text { si } x>0)
$$

(*) Voir Kит.1.вsck (8), page 102 . 
en approximant $\log \mathrm{a} / \mathrm{b}$ par la moyenne des deux bornes - approximation qui est d'autant meilleure que $a / b$ est proche de 1

on peut écrire :

$$
\mathrm{a} \# \mathbf{b}
$$

$$
\log a / b \sim \frac{a^{2}-b^{2}}{2 a b}
$$

$$
I\left(*: 2 ; 0_{N}\right)=\sum_{1=1}^{c} \quad x_{1} \log \frac{x_{1}}{N p_{1}}
$$$$
\sum_{1} x_{1} \cdot \frac{x_{1}^{2}-N^{2} p_{1}^{2}}{2 x_{1} N p_{1}}=\frac{1}{2} \quad \sum_{1} \frac{\left(x_{1}-N p_{1}\right)^{2}}{N p_{1}}
$$$$
2 I\left(*: 2 ; 0_{N}\right) \sim \sum \frac{\left(x_{1}-N p_{1}\right)^{2}}{N p_{1}}
$$

On retrouve au second membre une expression familière qui suit une loi de $\chi^{2}$ à $(c-1)$ degrés de liberté. 
DEUXIÈME PARTIE

\section{APPLICATIONS PRATIQUES}

0. - Nous nous proposons maintenant d'exposer un certain nombre d'applications pratiques de l'information. Le sujet est vaste et nous nous limiterons volontairement au cadre restreint de la loi multinomiale qui permet la comparaison d'échantillons et l'étude des tables de contingence. Il sera possible par la suite, de développer ou de prolonger cette étude dans d'autres domaines sans qu'il soit nécessaire de revenir sur les principes théoriques de base qui ont été exposés dans la première partie.

\section{Remarques préliminaires}

0. 1 - La définition de l'information, de même que celle de l'information minima de discrimination 1 ou de son estimation I sont valables dans le cas le plus général. La densité de probabilité généralisée dont la définition résulte de l'application du théorème de RADON-NYKoDYM n'a fait l'objet d'aucune restriction (hormis l'existence d'une mesure de probabilité dominante différente de zéro). A aucun moment, on n'a, en particulier, supposé que la densité de probabilité était celle de la loi normale (à une ou plusieurs dimensions). Cependant, les événements ou les suites d'événements considérés sont toujours aléatoires, ce qui exclut en particulier, tout abandon de la randomisation dans les dispositifs où l'on se proposera d'estimer un paramètre (ou l'effet d'un facteur contrôlé).

0.2 - La convergence asymptotique de $2 \mathrm{I}\left(*: 2 ; \mathrm{ON}_{\mathrm{N}}\right)$ vers la loi de $\chi^{2}$ est également démontrée de façon générale: la démonstration utilise le théorème central limite et implique que $\theta$ est de la forme $1 / \mathrm{n}$ fois la somme de $\mathrm{n}$ vecteurs indépendants ( $\theta$ est géné- 
ralement multidinensionnel) ayant une matrice de covariance finie. La convergence sera d'autant mietx assurée que le nombre d'olservations sera élevé.

Cependant, l'estimation de l'information minima de discrimination demeure une donnée valable pour apprécier la ressemblance de detux populations $\left({ }^{\circ}\right)$, indépendamment de son comportement asymptotique. Elle pourra toujours servir à discriminer plusieurs hypothèses (cf, paragraphe 4.2 ci-dessus) et à discerner la plus vraisemblable.

0. 3 - L'information fait intervenir des logarithmes népériens: on verra plus loin qu'il n'en résulte aucune complication particulière car il existe des tables de $n \log _{n} n$ étendues et très précises. On trouvera en particulier dans KuLlasck (8), p. 367 et sq. des tables donnant la valeur de $n \log n$ avec dix décimales pour $n$ compris entre 1 et 1000 .

Une table moins étendue, calculée sur la CAE 510 du Centre de Calcul Automatique de l'Université de Nancy, figure en annexe. Sit précision est largement suffisante pour les calculs courants. Pour les nombres ne figurant pas sur la table, on peut toujours utiliser la formule

$$
\log _{e} n=2,3026 \log _{10} n
$$

permettant de passer des logarithmes décimaux aux logarithmes népériens.

\section{1 - Comparaison d'échantillons de populations multinomiales}

1, 0 - Rappelons brièvement que, pour un seul échantillon, il est possible de tester une hypothèse nulle $\mathrm{H}_{2}$ : l'échantillon

$$
\left(x_{1}, x_{2} \ldots x_{0}\right), \quad \mathrm{z}_{1} x_{1}=N
$$

appartient à la poptulation multinomiale dont les fréquences de classes sont $p_{1}, \Sigma_{p_{i}}=1 \quad i-1.2$... c. par:

$$
\begin{aligned}
&\left.\hat{I}(*: 2) o_{N}\right)= \sum_{1} x_{1} \log \frac{x_{1}}{N p_{1}} \\
& \text { formule (4. 4) paragraphe 1-4.1 } \\
&=\sum_{1} x_{1} \log x_{1}-\sum_{1} x_{1} \log p_{1}-N \log N
\end{aligned}
$$

(*) Ou de deux hypothèses caractérisées par leur loi de répartition $f_{1}(x), f_{2}(x)$. 
$2 \hat{\mathrm{I}}\left(*: 2 ; \mathrm{ON}_{\mathrm{N}}\right)$ est asymptotiquement distribuée comme $\chi^{2}$ avec (c - 1) degrés de liberté dans l'hypothèse nulle, ce qui permet de déterminer un intervalle de confiance pour $2 \mathrm{I}\left({ }^{*}, \mathrm{p}\right)$; si la valeur observée $2 \mathrm{I}\left(\right.$ * : $2 ;$ ON) calculée à partir des $x_{1}$ observés par (4.4) est inférieure à $\chi^{2} 0,05$ pour $v-c-1$ degrés de liberté l'hypothèse nulle peut être acceptée.

On montre encore que $I\left({ }^{*}: 2 ; \mathrm{ON}_{\mathrm{N}}\right)$ peut être décomposé de façon additive pour tester l'opportunité de certains regroupements de classes que l'examen des données peut suggérer. Nous laisserons ce problème de cóté pour nous intéresser plus spécialement à la comparaison de deux ou plusieurs échantillons.

\section{1 - Comparaison de deur ćchantillons.}

Le probleme est le suivant: on dispose de deux ćchantillons independants dont les éléments sont répartis en c classes d'effectifs:

$$
\begin{array}{lll}
x_{1}, x_{2}, \ldots, & x_{1} \ldots, x_{e} & \Sigma_{i} x_{1}=N_{1} \text { pour l'échantillon } 1 \\
y_{1}, y_{2}, \ldots & y_{1} \ldots, y_{e} & \Sigma_{i} y_{1}=N_{2} \text { pour l'échantillon } 2
\end{array}
$$

Peut-on admettre que ces deux échantillons sont extraits d'une même population c-multinomiale?

On testera une hypotèse alternative $H_{1}$ : les échantillons appartiennent à deux populations distinctes, caractérisées par les probabilités

$$
\mathrm{P}_{1}:\left(\mathrm{P}_{11}, \mathrm{P}_{12}, \ldots \mathrm{P}_{1}, \ldots \mathrm{P}_{\mathrm{e}}\right) \quad \boldsymbol{\Sigma}_{1} \mathrm{P}_{11}-1
$$

$$
\mathrm{P}_{2}:\left(\mathrm{P}_{21}, \mathrm{P}_{22}, \ldots \mathrm{P}_{21}, \ldots \mathrm{P}_{2 c}\right) \quad \mathbf{\Sigma}_{1} \mathrm{p}_{21}=1
$$

contre l'hypothèse nulle $\mathrm{H}_{2}$ : les échantillons appartiennent à une seule population caractérisée par les probabilités:

$$
\mathrm{p}:\left(\mathrm{p}_{1}, \mathrm{p}_{2} \ldots \mathrm{p}_{1} \ldots, \mathrm{p}_{\mathrm{e}}\right) \quad \mathbf{\Sigma}_{1} \mathrm{p}_{1}=1
$$

En vertu de la propriété d'additivité de l'information (cf. paragraphe $1-2.1)$ et de l'indépendance mutuelle des échantillons.

$$
I(1 ; 2)=N_{1} \sum_{1} p_{11} \log \frac{p_{11}}{p_{1}}+N_{2} \quad \sum_{1} p_{21} \log \frac{p_{21}}{p_{1}}
$$


Les distributions conjuguées sont — voir exemple du paragraphe I - 3. 2 .

$$
\mathrm{p}_{1}(\mathbf{x})=\frac{\mathrm{p}(\mathrm{x}) \exp \left(\tau_{11} \mathrm{x}_{1}+\cdots \tau_{1 c} \mathrm{x}_{c}\right)}{\mathrm{A}_{1}} \text { où } A_{1}=\sum_{1} p_{1} \exp \left(\tau_{11} \mathbf{x}_{1}\right)
$$

$$
p_{2}^{*}(x)=\frac{p(x) \exp \left(\tau_{21} x_{1}+\cdots \tau_{2 c} x_{c}\right)}{A_{2}} \quad A_{2}=\sum_{1} p_{1} \exp \left(\tau_{21} y_{1}\right)
$$

$$
\begin{array}{r}
I(* ; 2)=I\left(p^{*}: p\right)-\sum_{1}\left(\left(\tau_{11} B_{1}^{*}\left(x_{1}\right)+\tau_{21} E_{2}^{*}\left(y_{1}\right)\right)\right. \\
-N_{1}\left(p_{1} e^{\tau_{1} 1}+\ldots p_{c} e^{\tau 1 c}\right)-N_{2}\left(p_{1} e^{\tau 21}+\ldots p_{c} e^{\tau 2 c}\right)
\end{array}
$$

par application de lit formule définissant $I(*: 2)$ (paragraphe I - 3. 1).

En posant

$$
E_{1}^{*}\left(x_{1}\right)=N_{1} P_{11}^{*} \quad E_{2}^{*}\left(y_{1}\right)=N_{2} P_{21}^{*}
$$

comme dans l'exemple du paragraphe $1-3,2$, on a:
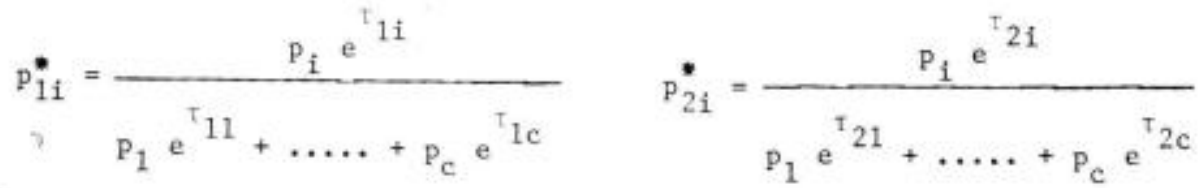

d'ou l'on tire les valeurs de $\tau 1$ et $\tau$ en et (1, 5) devient (1. 6)

$$
I\left(p^{*}: p\right)=N_{1} \sum p_{11}^{*} \log \frac{p_{11}}{p_{1}}+N_{2} \sum p_{21}^{*} \log \frac{p_{21}^{*}}{p_{1}}
$$


L'estimation $\mathrm{I}_{\left(\mathrm{p}^{*}: \mathrm{p}\right)}$ de $\mathrm{I}\left(\mathrm{p}^{*}: \mathrm{p}\right)$ fait appel aux meilleurs estimateurs sans biais de $\mathrm{p}^{*}{ }_{11}$ et de $\mathrm{p}^{*}{ }_{21}$ qui sont les fréquences relatives observées:

$$
\begin{aligned}
& \hat{p}_{11}^{*}=\frac{x_{1}}{N_{1}} \quad \hat{p}_{21}^{*}=\frac{y_{1}}{N_{2}} \quad \text { d'où } \\
& \hat{I}(p *: p)=N_{1} \sum_{1} \frac{x_{1}}{N_{1}} \log \frac{x_{1}}{N_{1} p_{1}}+N_{2} \sum_{1} \frac{y_{1}}{N_{2}} \log \frac{y_{1}}{N_{2} p_{1}}
\end{aligned}
$$

$$
\hat{I}\left(p^{*}: p\right)=\Sigma_{1}\left(x_{1} \log \frac{x_{1}}{N_{1} p_{1}}+y_{1} \log \frac{y_{1}}{N_{2} p_{1}}\right)
$$

L'estimation de l'information minima de discrimination que nous venons de calculer est relative à une hypothèse nulle spécifiant les valeurs $p_{1}$ des probabilités de classe commune aux deux échantillons. Le test de $\mathrm{I}\left(\mathrm{p}^{*}: \mathrm{p}\right)$ dépasse par conséquent le but que nous nous sommes assigné qui se linite au test de l'homogénéité des échantillons; en effet, une valeur de $I\left(p^{*}: p\right)$ non significative autorise à ne pas rejeter les hypothèses simultanées: $\mathrm{H}_{2}($.$) ;$

- les échantillons en premier lieu sont homogènes - hypothèse

ils appartiennent en outre à une population dont les probabilités de classe sont les valeurs de $p_{i}$ adoptées dans l'expression (1. 7) - hypothèse $\mathrm{H}_{2}(. / \mathrm{p})$. L'hypothèse $\mathrm{H}_{2}$ de la formule (1.2) est ainsi l'intersection de deux hypothèses:

$$
\mathrm{H}_{2}=\mathrm{H}_{2}(.) \cap \mathrm{H}_{2}(. / \mathrm{p})
$$

L'information $\mathrm{I}\left(\mathrm{p}^{*}\right.$ : $\left.\mathrm{p}\right)$ peut être additivement décomposée pour tester chacune de ces hypothèses: un premier terme permet de tester les meilleures estimations sans biais $p_{1}$ :

$$
\hat{p}_{1}=\frac{x_{1}+y_{1}}{N_{1}+N_{2}}
$$

des probabilités de classe (à partir des deux échantillons groupés) par rapport aux $p_{1}$; le second terme, analogue à un terme d'erreur, permet de tester l'homogénéité des échantillons à l'égard de cette estimation commune.

$$
\text { A } \begin{aligned}
& \mathrm{H}_{2}=\mathrm{H}_{2}(\cdot) \cap \mathrm{H}_{2}(* / p) \text { correspond } \\
& \hat{I}\left(p^{*}: p\right)=\hat{I}\left(p^{*}: \hat{p}\right)+\hat{I}(\hat{p}: p)
\end{aligned}
$$


En explicitant les valeurs correspondantes, on obtient le tableat d'analyse suivant, ou $\Sigma_{1}$ désigne la sommation de $\mathrm{i}=1$ à $\mathrm{i}=\mathrm{c}$

\begin{tabular}{|c|c|c|}
\hline $\begin{array}{l}\text { Composante } \\
\text { due in }\end{array}$ & Informations & $\begin{array}{l}\text { degrés de } \\
\text { liberté }\end{array}$ \\
\hline $\begin{array}{l}2 \hat{I}(\hat{p} ; p) \\
2 \hat{I}\left(p^{*}: p\right)\end{array}$ & $\begin{array}{l}2 I_{1}\left(x_{1}+y_{1}\right) \log \frac{x_{1}+y_{1}}{\left(N_{2}+N_{2}\right) D_{1}} \\
2 E_{1}\left(x_{1} \log \frac{\left(N_{1}+N_{2}\right) x_{1}}{N_{1}\left(x_{1}+y_{1}\right)}+y_{1} \log \frac{\left(N_{1}+N_{2}\right) y_{1}}{N_{2}\left(x_{1}+y_{1}\right)}\right)\end{array}$ & $\begin{array}{l}c=1 \\
c-1\end{array}$ \\
\hline $2 \hat{I}\left(p^{*}: D\right)$ & $2 I_{1} x_{1} \log \frac{x_{1}}{N_{1} p_{1}}+y_{1} \log \frac{y_{1}}{N_{2} p_{1}}$ & $2(c-1)$ \\
\hline
\end{tabular}

Tableau 1.1 - Analyse de finfermation (2 échantillons)

La colonne "degrés de liberté o indique ceux de la distribution de $\chi^{2}$ vers laquelle tendent asymptotiquement chacune de ces expressions daus thypothèse unlle. L'expression de $\mathrm{I}\left(\mathrm{p}^{*}: \mathrm{p}\right)$ n'est autre que celle de la formule (1.7) où les probabilités $p_{1}$ sont remplacées pat leurs estimations groupées:

$$
\hat{\mathrm{p}}_{1}=\frac{\mathrm{x}_{1}+\mathrm{y}_{1}}{\mathrm{~N}_{1}+\mathrm{N}_{2}}
$$

Le test d'homogénéité se fait en comparant la valeur de $2 \hat{\mathrm{I}}\left(\mathrm{p}^{*}: \mathrm{p}\right)$ à la valeur de $\gamma^{2}$ significative pour $c-1$ degrés de liberté. En développant, il vient :

$$
\begin{aligned}
\mathrm{I}\left(\mathrm{p}^{*}: \mathrm{p}\right) & =\mathbf{\Sigma}_{1}\left(\mathrm{x}_{i} \log \mathrm{x}_{i}+y_{1} \log y_{i}\right)+\mathrm{N} \log \mathrm{N} \\
& -\mathbf{\Sigma}_{i}\left(\mathrm{x}_{1}+y_{i}\right) \log \left(\mathbf{x}_{i}+\mathrm{y}_{i}\right)-\mathrm{N}_{1} \log \mathrm{N}_{1}-\mathrm{N}_{2} \log \mathrm{N}_{2}
\end{aligned}
$$

ivec $\mathrm{N}_{1}+\mathrm{N}_{2}=\mathrm{N}$.

Tous les termes sont de la forme $\mathrm{n} \log \mathrm{n}$ et peuvent être facilement calculés dès lors qu'on dispose d'une table $\left({ }^{*}\right)$.

(*) En ce qui concerne les valeurs significatives de $\chi^{2}$ la plupart des ouvrages de statistique, par exemple Vesseread (12), renferment des tables donnant ces valeurs aux seuils de $5 \%$ et de $1 \%$ au moins jusqu'à 30 degrés de liberté. Au delà, on peut utiliser la table, également classique, des probabilités cumulées de la loi normale en considérant que la variable $\sqrt{2 \chi^{2}}-\sqrt{2 v-1}$ suit approximativement la loi normale réduite. On aura toutefois intérèt à consulter des tahles plus complètes, par exemple dans SNédécor (10, pp. 28-29). 


\section{Exemple d'application.}

Pour une expérience destinée à étudier les possibilités de destruction sur pied du charme par badigeonnage à hauteur d'homme avec une solution de 2.4.5-T dans le fuel domestique, on a sélectionné 144 tiges qui ont été traitées en 1962 à trois époques différentes (symbolisées par $\mathrm{A}, \mathrm{P}$ et $\mathrm{H}$ ) et à det1x concentrations (1 et 2), l'attribution des traitement étant faite au hasard sur des lots de mème effectif. L.es résultats unt été observés en 1965 en attribuant à chaque arbre une a note o choisie dans une échelle de 0 à 6 - 0 correspondant à l'arbre mort et 6 à l'arbre indenne. Ces observations sont condensées dans le tableau 1, 2 (il n'v a pas d'arbres notés 6).

\begin{tabular}{|c|c|c|c|c|c|c|c|c|}
\hline Epoque & Dose & 0 & 1 & 2 & 3 & 4 & 5 & Total \\
\hline \multirow{3}{*}{ A } & 1 & 10 & 1 & 3 & 3 & 2 & 5 & 24 \\
\hline & 2 & 10 & 0 & 3 & 2 & 5 & 4 & 24 \\
\hline & Total & 20 & 1 & 6 & 5 & 7 & 9 & 48 \\
\hline \multirow{3}{*}{$P$} & 1 & 8 & 1 & 1 & 8 & 3 & 3 & 24 \\
\hline & 2 & 11 & 1 & 1 & 4 & 2 & 5 & 24 \\
\hline & Total & 19 & 2 & 2 & 12 & 5 & 8 & 48 \\
\hline \multirow{3}{*}{$\mathrm{H}$} & 1 & 3 & 0 & 1 & 3 & B & 9 & 24 \\
\hline & 2 & 6 & 1 & 0 & 1 & 9 & 7 & 24 \\
\hline & Total & 9 & 1 & 1 & 4 & 17 & 16 & 48 \\
\hline \multicolumn{2}{|c|}{ Total $(A+P+H)$} & 48 & 4 & 9 & 21 & 29 & 33 & 144 \\
\hline
\end{tabular}

Tableau 1.2 - Traitement badigeonnage 2.4.5-T - 1962

A titre d'exemple de la comparaison de deux échantillons, nous prendrons celle qui concerne les deux doses de l'époque $\mathrm{H}$ - dont la raison apparaitra plus loin.

On a ici $\mathrm{N}_{1}-\mathrm{N}_{2}=24$. Les valeurs de $\mathrm{x}_{1}+y_{\mathrm{i}}$ figurent sur la ligne " total " de la case $\mathrm{H}: 9,1,1,4,17,16$

$$
\mathrm{N}_{1}+\mathrm{N}_{2}-\mathrm{N}=48
$$


d'ou (en tenant compte que $0 \log 0=0$ ):

$$
\begin{array}{rr}
\Sigma_{1} x_{1} \log x_{1}+\Sigma_{1} y_{1} \log y_{1} & =87,1491 \\
+N \log N & +185,8176 \\
-\Sigma_{1}\left(x_{1}+y_{1}\right) \log \left(x_{1}+y_{1}\right) & -117,8462 \\
-N_{1} \log N_{1}-N_{2} \log N_{2} & \hat{I}=\frac{152,5466}{2,5739}
\end{array}
$$

$2 \mathrm{I}\left(\mathrm{p}^{*}: \mathrm{p}\right)-5,15$ est asymptotiquement distribuée comme $\chi^{2}$ avec $c-1-5$ degrés de liberté. Alors:

$$
0,25<\text { Prob. }\left(\chi^{2}=5,15\right)<0,50
$$

Bien que le nombre d'observations ne soit pas très élevé $(N=48)$. on peut penser qu'il existe une marge de sécurité suffisante pour affirmer que les deux distributions (done les résultats des traitements) ne diffèrent pas significativement.

Remarque: Il n'est pas inutile de rappeler que c'est 2 I (et non I) qui suit la distribution de $\chi^{2}$ : il ne faut done pas omettre de multiplier par 2 la valeur trouvée pour 1 , une telle omission pouvant évidemment modifier radicalement les conclusions du test.

\section{2 - Comparaison de plusicurs ichantillons.}

Nous voulons maintenant comparer $r$ échantillons afin de connaitre s'ils peuvent appartenir à une mème population multinomiale et, dans la négative, s'il existe des échantillons ou des grompes d'échantillons appartenant ì des poptlations distinctes.

L'échantillon d'ordre $i, 1 \leqslant i \leqslant r$, sera défini par:

$$
\left(x_{1}\right): x_{11}, x_{12}, \ldots x_{1 j}, \ldots, x_{1 c} \quad 1 \leqslant j \leqslant c
$$

Nous poserons:

$$
\Sigma_{j} x_{1 j}=N_{1} \quad \Sigma_{i} x_{1 j}=x_{j} \quad \Sigma_{1} N_{1}=N=\Sigma_{j} x_{j}
$$

\section{21 - Analyse globale de thomogénéité.}

Deux hypothèses peuvent être formulées:

$\mathrm{H}_{1}$ : les $\mathrm{r}$ échantillons appartiennent à des populations multinomiales distinctes dont les probabilités sont respectivement:

$$
p_{1}:\left(p_{11}, p_{12}, \ldots, p_{1 c}\right) \quad 1=1,2 \ldots r
$$


$\mathrm{H}_{z}$ : les $\mathrm{r}$ échantillons appartiennent à une population unique caractérisée par $\mathrm{p}:\left(\mathrm{p}_{1}, \mathrm{p}_{2} \ldots \mathrm{p}_{\mathrm{c}}\right)$, c'est-à-dire:

$$
p_{i 1}-p_{j} \quad \text { tout } i, 1 \leqslant i \leqslant r: \quad 1 \leqslant j \leqslant c
$$

Un raisonnement analogue à celui du paragraphe 1,1 précédent montre que:

$$
I(1: 2)=\Sigma_{1} N_{1} \sum_{j} p_{1 j} \log \frac{p_{1 j}}{p j}
$$

(1. 10)

$$
\hat{I}\left(p^{*}: p\right)=\Sigma_{1} \Sigma_{j} x_{1 j} \log \frac{x_{1 j}}{N_{j}}
$$

De la même façon l'estimation $\mathrm{I}\left(\mathrm{p}^{*}: \mathrm{p}\right)$ peut être décomposée additivement en deux termes: $I(p: p)$, relatif aux écarts entre les $p_{j}$ et la meilleure estimation sans biais à partir des $r$ échantillons groupés et $\mathrm{I}\left(\mathrm{p}^{2}\right.$ : $\left.\mathrm{p}\right)$ relatif aux déviations par rapport à $\mathrm{p}$ à l'intérieur des échantillons. Ces deux estimations suivent asymptotiquement une distribution de $\chi^{2}$ avec respectivement $c-1$ et $(\mathrm{r}-1)(\mathrm{c}-1)$ degrés de liberté. Le test d'homogénéité des échantillons se fait en comparant la valeur de $2 \mathrm{I}\left(\mathrm{p}^{*}: \mathrm{p}\right)$ à la valeur de $\chi^{2}$ significative ati seuil de probabilité choisi.

D'où le tableau d'analyse qui suit:

\begin{tabular}{|c|c|c|}
\hline Composante due à & Information & Degrés de l1berté \\
\hline $2 \hat{I}(\hat{p}: p)$ & $2 \Sigma_{j} x_{j} \log \frac{x_{j}}{N_{p}}$ & $c-1$ \\
$2 \hat{I}\left(p^{*}: \hat{p}\right)$ & $2 \sum_{1} \sum_{j} x_{1 j} \log \frac{N_{1 j}}{N_{1} x_{j}}$ & $(r-1)(c-1)$ \\
\hline $2 \hat{I}\left(p^{*}: p\right)$ & $2 \sum_{1} \Sigma_{j} x_{1 j} \log \frac{x_{1 j}}{N_{j}}$ & $r(c-1)$ \\
\hline
\end{tabular}

Tableau $1.3-\mathrm{r}$ échantillons - Analyse globale.

où les sommations sont étendues de 1 à $\mathrm{r}$ pour $\mathrm{i}$ et de 1 à $\mathrm{c}$ pour $\mathrm{j}$. 
Le tableau ci-dessts exprime, avec les mènes notations qu'au paragraphe 1, 1, que

$$
\mathrm{H}_{2}=\mathrm{H}_{2}(\cdot) \cap \mathrm{H}_{2}(\cdot \mathrm{p})
$$

La valeur qui nous intérese $2 \mathrm{I}\left(\mathrm{p}^{*}\right.$ : $\left.\mathrm{p}\right)$ se développe encore en $n$ log $n$ et petut s'écrire:

$2 \hat{I}\left(\hat{p}: p^{*}\right)=2 \Sigma_{1} \Sigma_{j} x_{1, j} \log x_{1 j}+2 N \log N-2 \Sigma_{j} x_{j} \log x_{j}$

$$
-2 \Sigma_{1} N_{1} \log N_{1}
$$

\section{Firemples d'application.}

\section{Extemple 1:}

Nous reprenons l'exemple cité dans l'untroduction. Le tableau des fréquences était le suivant:

\begin{tabular}{|c|c|c|c|c|c|c|c|c|}
\hline Traitement & 0 & 1 & 2 & 3 & 4 & 5 & 6 & Total \\
\hline T1 & 0 & 0 & 1 & 2 & 20 & 17 & 0 & 40 \\
T2 & 1 & 0 & 0 & 2 & 13 & 23 & 1 & 40 \\
T3 & 2 & 2 & 5 & 6 & 17 & 8 & 0 & 40 \\
\hline Total $\left(x_{f}\right)$ & 3 & 2 & 6 & 10 & 50 & 48 & 1 & $N-120$ \\
\hline
\end{tabular}

Tableau 1.4 - Traitements chimiques elagage, 1962

Les totaux de lignes et de colonnes sont respectivement les $\mathrm{N}_{\text {}}$ et les $x_{3}$ de la formule (1. 11). La table $n \log n$ doit donc ètre consultée pour toutes les valeurs intérieures et marginales du tableau. On trouve ici.

$$
\begin{aligned}
& \sum \sum x_{1 j} \log x_{1 j} \\
+ & N \log N \\
- & \sum N_{1} \log N_{1} \\
- & \sum x_{j} \log x_{j} \\
\hline & \hat{I}\left(\hat{p}: p^{*}\right)
\end{aligned}
$$

$$
\begin{array}{r}
302,6830 \\
+\quad 574,4990 \\
-\quad 442,6656 \\
-\quad 419,8774 \\
\hline 14,6390
\end{array}
$$


$2 \mathrm{I}=29,28$ est asymptotiquement distribuée comme $\chi^{2}$ avec $2 \times 6=12$ degrés de liberté $(\mathrm{r}=3, \mathrm{c}=7$ ).

Comme $\chi^{2}{ }_{0,05}-21,03$ et $\chi^{2}{ }_{0.01}=26,22$, on peut conclure que les traitements diffèrent significativement. I'examen du tableau suggère que $T_{3}$ diffère probablement de $T_{1}$ et $T_{2}$. Nous verrons qu'il est possible de tester cette hypothèse en décomposant $2 \mathrm{I}\left(\mathrm{p} ; \mathrm{p}^{*}\right)$.

\section{Exemple 2:}

En reprenant les données du tablesu 1, 2, on trouve pour l'analyse glohale des 6 échantillons ( 3 époques $\times 2$ concentrations):

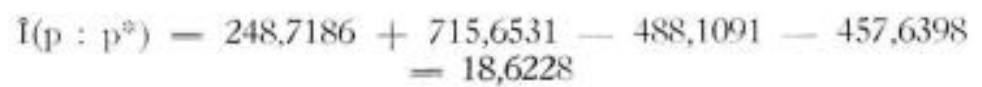

$2 I=37,25$ est distribuée asymptotiquement comme $\chi^{2}$ avec $5 \times 5-25$ degrés de liberté, Ia valeur limite au seuil $5 \%$ est alors: $\chi_{0.05}^{2}=37,65$.

Il y a peu de chances pour que les distributions soient homogènes et il serait intéressant de disposer d'une analyse plus fine en disséquant davantage ces échantillons, ce qui est possible comme on va le voir.

\section{22 - Analyse progressioc de lhomogénéité de différents ichantillons.}

On démontre que la loi de distribution de deux échantillons appartenant à une mème loi multinoniale peut être considérée comme le produit de la loi de distribution multinomiale de l'échantillon unique (constitué par l'ensemble des deux précédents) par la loi de distribution conditionnelle de ceux-ci, étant donné l'échantillon regroupé (\%).

Cette propriété s'étend de proche en proche et permet la décomposition de l'hypothèse d'homogénéité en un produit (ou mieux

(*) Tl suffit d'expliciter les probabilité, $p\left(x_{1}, x_{2}=p\left(x_{1}\right) \cdot p\left(x_{2}\right)\right.$ et $\mathrm{p}\left(\mathrm{x}_{1}+\mathrm{x}_{. .}\right)$des échantillons:

$$
\begin{aligned}
& x_{1}:\left(x_{11}, x_{12} \ldots x_{1 c}\right) \quad x_{2}:\left(x_{21}, x_{22} \ldots x_{2 c}\right) \\
& \left(x_{1}+x_{2}\right):\left(x_{11}+x_{21}, x_{12}+x_{22}, \ldots, x_{1 c}+x_{2 c}\right.
\end{aligned}
$$

par lat formule dommant les prohathilités de la bi multinomiale. On voit alors que :

$$
\left.p\left(x_{1}+x_{2}\right)=p\left(x_{1}\right) p\left(x_{2}\right)=p\left(x_{1}+x_{2}\right) \cdot \hat{f}\left(x_{1}, x_{2}\right)\left(x_{1}+x_{2}\right)\right)
$$


intersection) d'hypothèses successives, conditionnées par les précédentes et impliquant le regroupement progressif des échantillons. On peut écrire, sous forme symbolique,

$\mathrm{H}_{2}=\mathrm{H}_{2}(1,2) \cap \mathrm{H}_{2}(1+2,3) \cap \ldots \quad \ldots \cap \mathrm{H}_{2}(1+2+\ldots+r-1, r)$

A cette cascade d'hypothèses correspond une dichotomie des informations puisque l'information est additive même en termes de probabilités conditionnelles. On a toujours (cf. paragraphe $\mathrm{I}-2,1$ )

$$
\mathrm{I}(1: 2 ; \mathrm{X}, \mathrm{Y})=\mathrm{I}(1: 2 ; \mathrm{X})+\mathrm{I}(1: 2 ; \mathrm{Y} / \mathrm{X})
$$

On peut done décomposer

$$
\hat{I}\left(p^{*}: \hat{p}\right)=\hat{I}\left(H_{1}: H_{2}\right)
$$

en ( $\mathrm{r}-1)$ termes, qui asymptotiquenent, sont distribués comme $\chi^{2}$ à $(c-1)$ degrés de liberté. Une telle analyse permet de mettre en évidence l'apparition d'un échantillon qui n'est pas homogène par rapport au groupe des précédents, d'où son intérêt dans certains cas.

Posons

$$
y_{1 j}=x_{1 j}+x_{2 j}+\ldots x_{1 j} \quad 2 \leqslant 1<r-1
$$

les $y_{i j}$ sont les sommes - pour chaque classe $\mathrm{j}$ - des éléments des $i$ premiers échantillons (totaux partiels a colonnes " jusqu’à la ligne i).

L'échantillon groupé a pour effectif total:

$$
\mathrm{y}_{11}+\mathrm{y}_{12}+\ldots \mathrm{y}_{1 c}=\mathrm{N}_{1}+\mathrm{N}_{2}+\ldots \mathrm{N}_{1}=\mathrm{M}_{1}
$$

La décomposition de $2 \mathrm{I}\left(\mathrm{p}^{*}: \mathrm{p}\right)$ s'effectue dans la forme indiquéc an tableat 1.5 .

A noter que chaque étape de l'analyse augmente de $(c-1)$ le nombre de degrés de liberté de la composante d'homogénéité par stite du regroupement progressif d'échantillons homogènes entre eux. Ce regroupement n'est évidemment plus justifié dès lors qu'apparait un échantillon $\mathrm{i}$ qui n'est plus homogène par rapport à l'ensemble de ses prédécesseurs $(1 \mathrm{a} i-1)$. Il faut faire alors appel à la partition par groupes d'échantillons. 


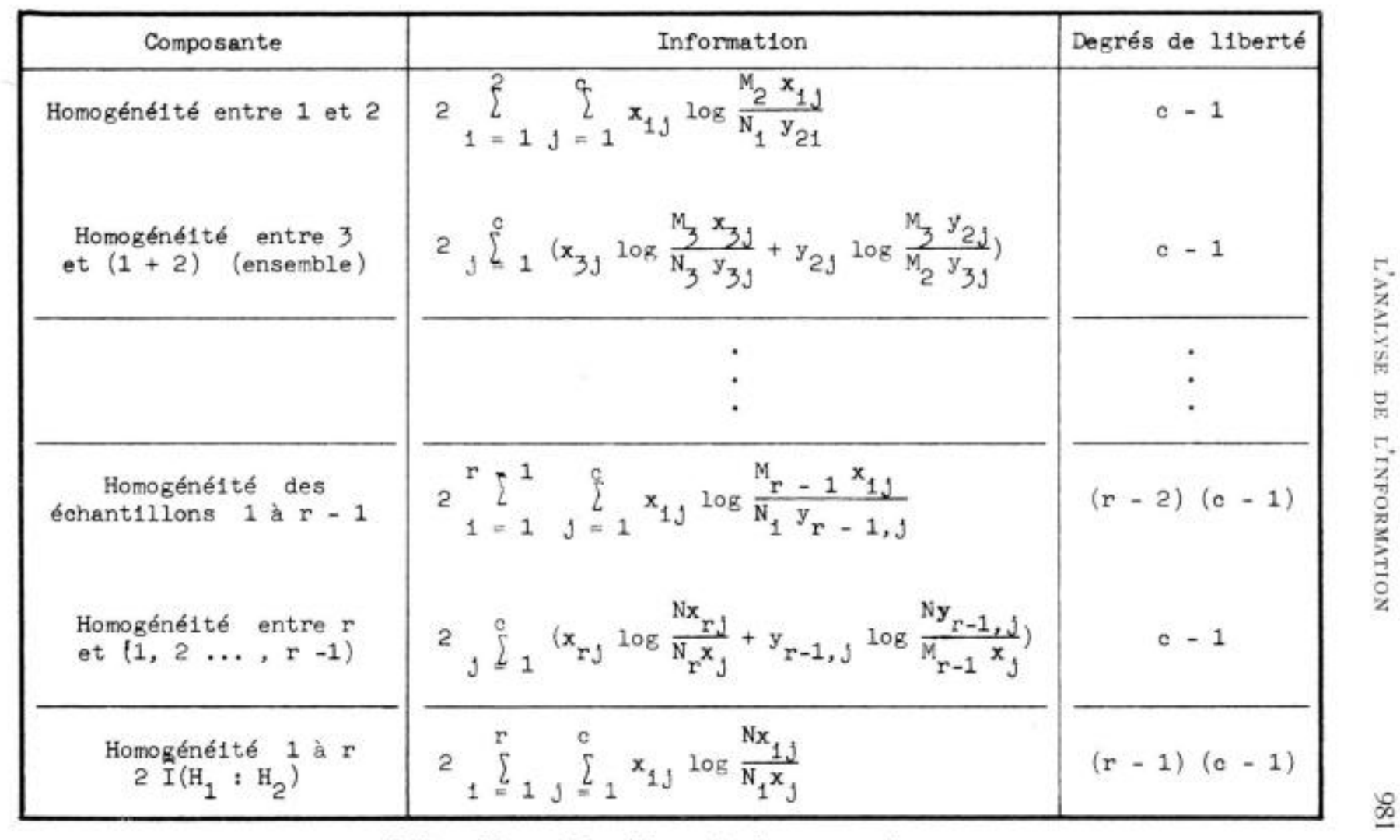

Tableau $1.5-\mathrm{r}$ échantillons - Analyse progressive. 
Comme précédenment, les termes de l'information sont tous de la forme $\mathrm{n} \log \mathrm{n}$ et les calculs ne présentent aucune difficulté lorsquion dispose d'une table donnant ces valeurs.

\section{Exemples d'application.}

\section{E.xemple 1:}

Nous poursuivons lanalyse des domnées de l'exemple 1 du paragraphe 1. 21 dont une précédente analyse a montré qu'ils n'étaient pas homogènes; comme l'examen des chiffres semble indiquer que les échantillons T1 et T2 le sont, nous commençons notre analyse progressive dans lordre du tableau par la comparaison T1: T2.

\begin{tabular}{|c|c|c|c|c|c|c|c|c|c|}
\hline Traitesient & Vartable & 0 & 1 & 2 & 3 & 4 & 5 & 6 & $N_{1}$ \\
\hline$T_{1}$ & $x_{1 j}$ & 0 & 0 & 1 & 2 & 20 & 17 & 0 & 40 \\
$T 2$ & $x_{2 j}$ & 1 & 0 & 0 & 2 & 13 & 23 & 1 & 40 \\
\hline$T 1+T 2$ & $y_{2 j}$ & 1 & 0 & 1 & 4 & 33 & 40 & 1 & $80-M_{2}$ \\
\hline
\end{tabular}

Tablean 1.6 - Analyse $\mathrm{T} 1: \mathrm{T} 2$

La comparaison détaillée des échantillons est ici simplifiée en raison de leur nombre: il suffira de calculer la composante d'homogénéité de T1 et T2 pour en déduire par différence la comparaison $(1+2): 3$; la somme des deux informations partielles est en effet égale à l'information $\mathrm{I}\left(\mathrm{H}_{1}: \mathrm{H}_{2}\right)-\mathrm{I}\left(\mathrm{p}^{*} ; \mathrm{p}\right)$ déjà calculée précédemment.

On peut écrire :

(1. 12)

$$
\begin{gathered}
\left.1 \sum_{1}^{2} x_{1 j} \log \frac{M_{2} x_{1 j}}{N_{1} y_{2 j}}=\sum_{1}^{2}=1\right\} x_{1 j} \log x_{1 j}+M_{2} \log M_{2} \\
-\sum_{1}^{2} N_{1} \log N_{1}-\sum, y_{2 j} \log y_{2 j}
\end{gathered}
$$

où $\Sigma_{j}$ indique la sommation de $\mathrm{j}=1 \mathrm{a} \mathrm{j}=\mathrm{c}$.

Les valeurs de $y_{2,}$ figurent au tableau 1, 6, par ailleurs;

$$
\begin{gathered}
\mathrm{N}_{1}=\mathrm{N}_{2}=40 \quad \mathrm{M}_{2}-\mathrm{N}_{1}+\mathrm{N}_{2}=80 \\
\mathrm{I}(1,2)=216,3125+350,5621-295,1104-268,4851 \\
=3.2791 \\
2 \mathrm{I}(1,2)=6,56
\end{gathered}
$$


Avec 6 degrés de liberté, on a $\chi_{0,0.5}^{2}-12,59$; la valeur trouvée pour 2 I n'est donc pas significative

$$
\text { - de fait } 0,25<\operatorname{Pr}\left(\chi^{2}-6,56\right)<0,50-
$$

et lon peut admettre que T1 et T2 constituent des échantillons homogènes entre eux.

La composante $2 \hat{\mathrm{I}}(1+2: 3)$ vaut, par différence (cf. paragraphe 1.21):

$$
\begin{aligned}
& 2 \mathrm{I}(1+2,3)=2 \mathrm{I}\left(\mathrm{p}^{t}: \mathrm{p}\right)-2 \mathrm{I}(1,2) \\
& \begin{array}{rrr}
-12,59 & -16,69 \\
\mathrm{dl}-6 & \chi^{2}{ }_{0.01}-16,81
\end{array}
\end{aligned}
$$

L'analyse de l'information indique done que le traitement $T_{3}$ donne des fréquences significativement différentes de celles données par les traitements $\mathrm{T} 1$ et $\mathrm{T} 2$, celles-ci pouvant être considérées comme homogènes entre elles.

\section{Remarque.}

Cet exemple a été volontairement choisi pour souligner les dangers de l'automatisme dans l'utilisation des méthodes statistiques. Le test d'homogénéité sur les échantillons T1 et T2 conduit à conclure que ces échantillons peuvent être considérés comme appartenant à une même distribution multinomiale dont les probabilités attachées à chaque classe ont pour valeur les fréquences relatives marginales $p_{j}=y_{2 j} / M_{2}$. On observe ici que $p_{1}=0$ (classe 1), ce qui signifie qu'en réalité la distribution commune ne comporte plus 7 , mais seulement 6 classes. Dans cette hypothèse, un test dhomogénéité plus précis peut être logiquement appliqué en abaissant de 6 à 5 le nombre de degrés de liberté du $\chi^{2}$ asymptotique mais on ne peut plus dès lors comparer des distributions dont le nombre de classe est différent suivant le choix des regroupements opérés,..

Si l'on observe dans une classe des effectifs nuls sur plusieurs échantillons et une fréquence très faible pour l'ensemble de ceuxci, on doit se demander si l'existence de cette classe est justifiée. Ia question se pose manifestement pour les données dt tableat 1.4 où l'on constate que les notes 1 et 6 sont absentes dans deux échantillons sur trois et que leur fréquence relative, pour l'ensemble, est respectivement $1 / 60$ et $1 / 120$. Ia définition de la notation conduit ici à admettre que l'on peut, compte tenu de la marge d'appréciation individuelle d'un observateur, regrouper les classes 1 et 2 et les classes 5 et 6 , ce qui évite d'avoir à affronter un problème théorique délicat. 


\section{Exemple 2:}

Sur les domnées du tableatu 1, 2 (paragraphe 1, 1), nous allons tester l'homogénéité des quatre preniers échantillons $\mathrm{A}, \mathrm{A2}, \mathrm{Pl}$. $\mathrm{P} 2$ et leur comparer le cinquiène (H1). Pour la clarté des caleuls, nous constituons le tableau suivant:

\begin{tabular}{|c|c|c|c|c|c|c|c|c|}
\hline Traiterrent & Variable & 0 & 1 & 2 & 3 & 4 & 5 & Totaux \\
\hline A $21=1$ & $x$ is & 10 & 1 & 3 & 3 & 2 & 5 & 24 (N1) \\
\hline$A \geq 1=2$ & $x^{51}$ & 10 & 0 & 3 & 2 & 5 & 4 & 24 (N2) \\
\hline P $11=3$ & $x_{31}$ & B & 1 & 1 & 8 & 3 & 3 & 24 (N3) \\
\hline P $21=4$ & $x_{49}$ & 11 & 1 & 1 & 4 & 2 & 5 & $24(\mathrm{~N} 4)$ \\
\hline$A 1+A 2+P 1+P 2$ & $y_{4 j}$ & 39 & 3 & 8 & 17 & 12 & 17 & $96(\mathrm{Na})$ \\
\hline H 1 - -5 & $x_{5 j}$ & 3 & 0 & 1 & y & B & 9 & 24 (N5) \\
\hline $\mathrm{A}_{1}+\mathrm{A}_{2}+\mathrm{P}_{1}+\mathrm{P}_{2}+\mathrm{H}_{1}$ & $y_{54}$ & 42 & 3 & 9 & 20 & 20 & 26 & 120 (N5) \\
\hline
\end{tabular}

Tableau 1.7

Nous calculons d'abord I(1 a 4 ), composante de l'information interne aux échantillons 1, 2, 3 et 4 de façon à tester l'homogénéité de cet ensemble. En développant, il vient:

$$
\begin{aligned}
\hat{I}(1 \text { à 4) } & =\sum_{1=1}^{4} \sum_{j} x_{1 j} \log x_{1 j}+M_{4} \log M_{4}-\sum_{1=1}^{4} N_{1} \log N_{1} \\
& -\sum_{j} y_{4, j} \log y_{4 j}
\end{aligned}
$$

Nors: On remarque que le calcul de $n \log n$ s'applique toujours aux valeurs internes et marginales du tableau constitué par le groupe d'échantillons étudié.

$$
\begin{aligned}
\hat{\mathrm{i}}(1 \mathrm{a} 4)-161,5695+ & 438,1774-305,0932-288,9583 \\
= & 5,6954
\end{aligned}
$$

$2 \mathrm{I}(1$ à 4$)=11,39$ est distribuée asymptotiquement comme $\chi^{2}$ avec $3 \times 5-15$ degrés de liherté, Ta probabilité d'observer la valeur trouvée est d'environ 0.75 car Prob $\left(\chi^{2}=11,04\right)=0,75$.

On peut, sans risque, conclure à l'homogénéité des quatre preniers échantillons.

L étape suivante consiste à calculer la seconde composante de l'information attachée à la comparaison de léchantillon 5 et du 
groupe $1+2+3+4$ considéré comme échantillon d'une distribution d'effectif total:

$$
\mathrm{N}_{1}+\mathrm{N}_{2}+\mathrm{N}_{4}+\mathrm{N}_{4}=\mathrm{M}_{4}
$$

On développe encore les différents termes de la formule dounant cette information, ce qui clonne, par mise en facteur des quantités non inclexées par $\mathrm{j}$ :

$$
\begin{aligned}
& \Sigma_{j} x_{5 j} \log x_{5 j}+\log M_{5} \cdot \Sigma_{j} x_{5 j}-\log N_{5} \cdot \Sigma_{j} x_{5 j} \\
& -\Sigma_{j} x_{5 j} \log y_{5 j}+\Sigma_{j} y_{4 j} \log y_{4 j}+\log M_{5} \cdot \Sigma y_{4 j} \\
& -\Sigma_{j} y_{4, j} \log y_{5 j}
\end{aligned}
$$

En tenant compte des égalités suivantes:

$$
\Sigma_{j} x_{5 j}=N_{5} \quad \Sigma_{j} y_{4 j}=M_{4} \quad M_{4}+N_{5}=M_{5}=\Sigma_{j} y_{5 j}
$$

il vient:

$$
\begin{aligned}
\hat{I} & =\Sigma_{j} y_{4 j} \log y_{4 j}+\Sigma_{j} x_{5 j} \log x_{5 j}+M_{5} \log M_{5}-M_{4} \log M_{4} \\
& -N_{5} \log N_{5}-\Sigma_{j} y_{5 j} \log y_{5 j}
\end{aligned}
$$

En définitive, on retrouve, une fois de plus, les valeurs internes et marginales du tableau constitué par l'échantillon groupé (1 à 4) et l'échantillon introduit daus le test d'homogénéité, règle dont lintérèt pratique est évident car elle évite de se reporter à des formules compliquées (en apparence).

Sur les chiffres du tableau 1, 7, on trouve:

$$
\imath-331.9604+574.4990-514.4507-384.5926=7.4161
$$

$2 \hat{\mathrm{I}}-14,83$ est distribuée comme $\chi^{2}$ avec $\mathrm{c}-1=5$ degrés de liberté. Cette valeur est significative car la probabilité de $\gamma^{2}=14,83$ est voisine de 0,01 (la valeur correspondant à 0,01 est 15,09 ). On conclut à l'existence d'une hétérogénéité significative entre l'échantillon 5 et les précédents. 


\section{23 - Comparaison de groupes d'échantillons.}

Dans l'exemple 1 qui précède, on aurait pu considérer que l'ensemble de trois échantillons pouvait constituer deux groupes: d'un cóté T1 et T2, de l'autre T3; plus généralement, il se peut que les conditions de l'expérimentation nécessitent - ou que l'examen des données suggère - des groupements possibles au sein de l'ensemble des échantillons. Ia question se pose alors de savoir si de tels groupements sont valables, c'est-à-dire de tester leur homogénéité interne et dans l'affirmative, d'examiner s'ils appartiennent à des distributions multinomiales distinctes. En analyse de variance. la méthode des contrastes peut permettre des jugements analogues. L'analyse de l'information autorise également de telles comparaison. grâce à la précieuse propriété d'additivité.

Soit encore $r$ échantillons $x_{i j} \quad 1 \leqslant i \leqslant r \quad 1 \leqslant j \leqslant c$

on suppose quion divise ces $r$ échantillons en deux groupes et que lon désire śassurer de la validité des regroupements.

Le groupe 1 est constitué des échantillons 1 à $\mathrm{k}$, le groupe 2 de $\mathrm{k}+1$ à $\mathrm{r}$. On pose alors:

$$
\begin{array}{ll}
z_{1 j}=x_{1 j}+x_{2 j}+\ldots x_{k j} & T_{1}=\Sigma_{j} z_{1 j} \\
z_{2 j}=x_{k}+1, j+\ldots x_{r J} & T_{2}=\Sigma_{j} z_{2 j}
\end{array}
$$

\begin{tabular}{|c|c|c|}
\hline Composante & Information & degré de 11 berté \\
\hline $\begin{array}{l}\text { Entre groupe } 1 \\
\text { et groupe } 2 \\
\text { Homogénétté du groupe } 1 \\
\text { Homogénéité du groupe } 2\end{array}$ & $\begin{array}{c}2 \Sigma_{j}\left(Z_{1 j} \log \frac{N Z_{1 j}}{T_{1} x_{j}}+Z_{2 j} \log \frac{N Z_{2 J}}{T_{2} x_{j}}\right. \\
2 \sum_{1=1}^{K} E_{j} x_{1 j} \log \frac{T_{1} x_{1 j}}{N_{1} Z_{1 j}} \\
2 \sum_{1=K+1}^{K} \Sigma_{j} x_{1, j} \log \frac{T_{2} x_{1 j}}{N_{1} Z_{2 J}}\end{array}$ & $\begin{array}{c}0-1 \\
(k-1)(c-1) \\
(k-k-1)(c-1)\end{array}$ \\
\hline $2 I\left(p^{*} ; \beta\right)=2 I\left(H_{1}: H_{2}\right)$ & $2 \sum_{1=1}^{r} \sum_{j} x_{1 J} \log \frac{N x_{1 j}}{N_{1} x_{j}}$ & $(x-1)(c-1)$ \\
\hline
\end{tabular}

La décomposition de l'information se fait alors comme suit:

Tablean $1.8-\mathrm{r}$ échantillons - Analyse de groupes.

Conme précédemment, le développement des termes des informations partielles se présente comme une suite de $n \log n$ et ne présente aucune difficulté. 


\section{Exemple d'application:}

Sur les données du tableau 1. 2 (paragraphe 1. 1), nous allons tester l'effet de la dose d'application en constituant deux groupes d'échantillons,

- d'une part, A1, A2, P1 et P2 (groupe 1)

- d'attre part, $\mathrm{H} 1$ et $\mathrm{H} 2 \quad$ (groupe 2)

ce qui donne les valeurs stivantes de $Z_{11}, Z_{21}, T_{1}$ et $T_{22}$ :

$$
\begin{array}{rrrr}
Z_{11}: 39,3,8,17,12,17 & T_{4}=96 \\
Z_{21}: 9,1,1,4,17,16 & T_{2}=48
\end{array}
$$

En développant les formules des composantes du tableau 1. 8, on trouve, par exemple, pour la composante interne au groupe 1

\section{(1. 13)}

$$
\sum_{1=1}^{k} \Sigma_{j} x_{1 j} \log x_{1 j}+T 1 \log T 1-\sum_{1=1}^{k} N_{1} \log N_{1}-\Sigma_{j} z_{1 j} \log z_{1 j}
$$

Là encore, ne subsistent au développement que les valeurs internes ou marginales du tableau partiel constitué par le groupe d'échantillons. De même pour la composante d'homogénéité entre groupes qui s'écrit:

$$
\begin{aligned}
& \Sigma_{j} z_{1 j} \log z_{1 j}+\Sigma_{j} z_{2 j} \log z_{2 j}+N \log N-\Sigma_{j} x_{j} \log x_{j} \\
& -T_{1} \log T_{1}-T_{2} \log T_{2}
\end{aligned}
$$

la règle habituelle s'applique en considérant comme valeurs internes les totaux partiels des deux groupes.

Les composantes internes à chaque groupe d'échantillons ont déjà été calculées précédemment (avec des notations légèrement différentes) aux paragraphes 1. 1, exemple d'application, et 1. 22, analyse progressive, exemple 2.

$$
\begin{array}{lll}
\text { On a trouvé } \mathrm{I}(1 \text { à } 4)=5,6954 & 2 \mathrm{I}(1 \text { à } 4)-11,39 \\
\mathrm{I}(5 \text { et } 6)=2,5739 & 2 \mathrm{I}(5 \text { et } 6)-5.15
\end{array}
$$

La comparaison des deux groupes donne:

$$
\begin{aligned}
\hat{I} & =406,8045+715,6531-488,1091-623,9950=10,3535 \\
2 \hat{I} & =20,71
\end{aligned}
$$


Le $\chi^{2}$ correspond ì c $-1=5$ degrés de liberté à une probabilité inférieure à 0.005 . I.es deux groupes diffèrent donc significativement.

En additionnant les trois valeurs trouvées pour l'information

$$
2\{5,6954+2,5739+10.3535\}=2 \times 18,6228
$$

on retrouve bien l'information totale qui a été calculée pour le test t'homogénéité globale de l'échantillon (paragraphe 1.21, exemple 2). Cette vérification permet de contröler les calculs: toutes les propriétés additives des cxpressions formelles de l'information découlent de l'additivité de la fonction logarithme, On pourrait encore décomposer l'homogénéité interne aux groupes 1 à 4 en deux composantes d'homogénéité interne à $(1+2)$ et $(3+4)$ et une composante d'homogénéité des deux groupes précédents. On voit combien la méthode est souple et permet de tester de nombreuses hypothèses.

Si nous récapitulons les résultats de lanalyse exhaustive des résultats du tableat 1, 2, on constate ce qui suit:

- l'ensemble des traitements riest pas homogène.

- l'ensemble des traitements A et P l'est, de même que l'ensemble H1, H2.

- il existe des différences significatives entre les deux grotnes ainsi définis.

On peut conclure, sur le plan pratique, que l'influence de la dose n'est pas significative et qu'en ce qui concerne la saison d'application, on obtient des résultats comparables au printemps et à lautomne (c'est-à-dire pendant la saison de végétation), ces résultats étant supérieurs à ceux que l'on observe avec un traitement d'hiver.

\section{Remarque 1:}

L'information se déconpose de façon additive suivant un schéma qui n'est pas sans analogie avec celui de l'analyse de variance — sans parler naturellement du $\chi^{2}$ pour lequel la ressemblance est encore plus marquée.

\section{Remarque 2:}

Il faut encore insister stur le fait qu'en dépit de la complexité apparente de la formulation des termes de l'information, les calculs se ramenent toujours à la sommation de termes en $\mathrm{n} \log \mathrm{n}$. Dans les cas simples, on pourra toujours utiliser une table; est-il nécessaire d'ajouter que le calcul des $\Sigma$ n log $n$ est un jeu pour le calculateur électronique qui pourra être mis à contribution dans les 
cas plus compliqués. Une règle simple s'applique aux sommations relatives au test d'homogénéité d'échantillons groupés: on calculera les valeurs de $\mathrm{n} \log \mathrm{n}$ pour les différents termes du tableau (intérieurs et marginaux) et leur signe découle du tableau suivant, qu'il est facile de retenir:

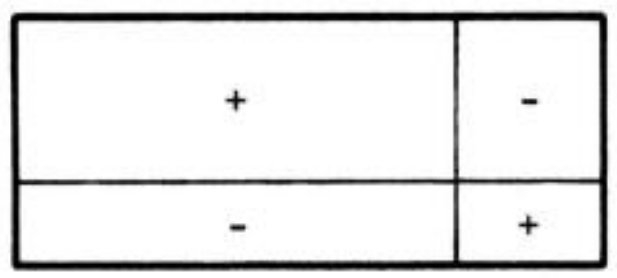

Par exemple:

$\hat{I}(1: 2)=\Sigma \Sigma x_{1 j} \log x_{1 j}+N \log N-\Sigma \Sigma_{j} x_{j} \log x_{j}-\sum_{1} N_{1} \log N_{1}$

\section{2 - Tables de contingence}

2. 0 - Une table de contingence est un échastillon d'une loi multinomiale dont les constituants sont classés suivant deux ou plusieurs critères. $\mathrm{Si}$ une table de contingence à double entrée $\mathrm{r} \times \mathrm{c}$ se présente formellement comme un tableat de comparaison de $r$ échantillons indépendants d'une loi multinomiale à c classes, il faut se garder de confondre les deux problèmes.

Dans la comparaison d'échantillons, les probabilités afférentes aux cases d'une même ligne ne sont pas indépendantes. Elles sont liées par la relation $\Sigma_{j} p_{1 j}=1$ ou encore $\Sigma_{j} x_{i j}=N_{i}$ effectif total choisi pour l'échantillon $\mathrm{i}$.

Le nombre de degrés de liberté total du $\chi^{2}$ asymptotique de l'estimation de l'information minima de discrimination $2 \mathrm{I}\left(\mathrm{p}^{*}: \mathrm{p}\right)$ est ainsi $\mathrm{r}(\mathrm{c}-1)$.

Dans une table de contingence à double entrée, le nombre de classes de la loi multinomiale n'est plus c, mais rc correspondant aux différentes combinaisons des deux caractères observés sur les $\mathrm{N}$ individus, L'effectif d'une ligne n'est plus fixé à priori: on le constate. Le nombre de degrés de liberté du $\chi^{2}$ asymptotique de l'estimation de l'information minima de discrimination

$$
2 \mathrm{I}\left[(\mathrm{p})^{*}:(\mathrm{p})\right] \text { est alors re }-1
$$

car on a une seule relation: $\boldsymbol{\Sigma}_{1} \boldsymbol{\Sigma}_{1} p_{1 j}-1$. 
A l'hypothèse d'homogénéité des échantillons se substitue une hypothèse d'indépendance des caractères définissant la classification en lignes et en colonnes. Cette distinction est un peu analogue à celle qui sépare, dans l'analyse de variance, le modèle fixe du modèle aléatoire (dans le modèle fixe, il est utile de comparer les moyennes, dans le modèle aléatoire cette comparaison est sans intérêt).

\section{1 - Tables de contingence à double cntrée.}

Soit une table de contingence à $r$ lignes et $\mathrm{c}$ colonnes dans laquelle on a classé les divers individus d'un échantillon d'effectif $\mathrm{N}$, l'effectif d'une case étant $x_{i j} \quad 1 \leqslant i \leqslant r \quad 1 \leqslant j \leqslant c$

Les fréquences marginales observées sont respectivement:

$$
\begin{aligned}
& x_{1 .}=\Sigma_{j} x_{1 j} \\
& \text { et } \Sigma_{1} x_{1 .}=\Sigma_{j} x_{\cdot j}=N
\end{aligned}
$$

L'hypothèse nulle est l'hypothèse d'indépendance des deux caractères quii, en termes de probabilités, s'écrit avec les mêmes indices:

$$
p_{11}=p_{1} \text {. P.s }
$$

Nous testerons donc l'hypothèse alternative $\mathrm{H}_{1}$.

$$
\begin{gathered}
H_{1}: p_{i j} \neq p_{i} \text {. P.s pour att moins un couple }(i, j) p_{i j}>0 \\
\Sigma_{1} \Sigma_{j} p_{1 j}-1
\end{gathered}
$$

contre l'hypothèse nulle,

$$
\mathrm{H}_{2}: \mathrm{p}_{1\}}=\mathrm{P}_{\mathrm{i}} \text {, P.s } \quad \forall \mathrm{i}, \mathrm{j}
$$

avec:

$$
\Sigma_{1} p_{1 .}=1 \quad \Sigma_{j} p_{. J}=1 \quad p_{1 .}>0 \quad p_{. J}>0
$$

On montre, comme dans le cas de la comparaison de $r$ échantillons, que 
(2. 1)

$$
I\left(H_{1}: H_{2}\right)=N \Sigma_{1} \Sigma_{j} p_{1 j} \log \frac{p_{1 j}}{p_{1} \cdot p_{j} \cdot j}
$$

Pour la distribution conjuguée $(\mathrm{p})^{2}$ dont les paramètres sont la meilleure estimation sans biais pouvant être obtenue à partir de l'échantillon, l'estimation $I\left[(p)^{*}:(p)\right]$ a pour valeur:

$$
\hat{I}(p) *:(p))=\Sigma_{1} \Sigma_{j} x_{1 j} \log \frac{x_{1 j}}{N_{1} \cdot P^{p} \cdot j}
$$

En pratique, nous ne voulons pas tester l'échantillon par rapport à l'hypothèse $\mathrm{H}_{2}$ qui implique des valeurs définies p. et $\mathrm{p}_{\text {.1 }}$ des distributions marginales. L'hypothèse nulle $\mathrm{H}_{2}$ dépasse donc l'objectif recherché: heureusement, elle peut encore être décomposée en plusieurs sous-hypothèses dont elle constitue l'intersection.

On peut en effet écrire:

$$
\mathrm{H}_{2}=\mathrm{H}_{2}(\mathrm{R}) \cap \mathrm{H}_{2}(\mathrm{C}) \cap \mathrm{H}_{2}(\mathrm{R} \times \mathrm{C})
$$

$\mathrm{H}_{2}$ (R) et $\mathrm{H}_{2}$ (C) spécifiant les valeurs $p_{i}$, et $\mathrm{p}_{. j}$ et $\mathrm{H}_{2}(\mathrm{R} \times \mathrm{C})$ exprimant l'indépendance sous ces conditions.

$L^{\prime}$ information $\mathrm{I}\left[(\mathrm{p})^{*}\right.$ : $\left.(\mathrm{p})\right]$ se décompose alors additivement en trois composantes qui correspondent respectivement:

- aux déviations entre les $\mathrm{p}$. et leurs estimations à partir de l'échantillon,

- aux déviations entre les P., et leurs estimations à partir de l'échantillon,

- aux déviations par rapport à l'hypothèse d'indépendance, compte tenu de l'estimation ci-dessus des distributions marginales.

On montre que les meilleures estimations des paramètres sont:

$$
\hat{p}_{1 .}=\frac{x_{1}}{N} \quad \hat{6}_{. j}=\frac{x^{\prime} \cdot j}{N} \quad \hat{p}_{1 j}=\frac{x_{1}}{N} \cdot \frac{x \cdot j}{N}
$$


et l'analyse $k$ de l'information se fait suivant le tableau ci-après:

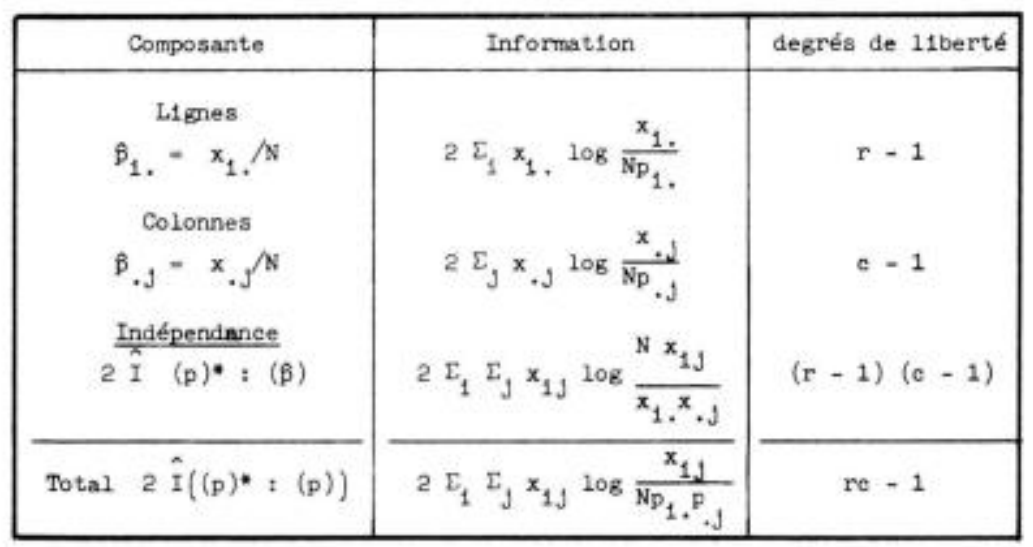

Tableau 2.1 - Indépendance, table à double entrée.

Le calcul de la composante d'indépendance est toujours aussi aisé avec la table de $n \log n$. On a en effet:

$$
\begin{aligned}
2 \hat{I}(p) *(\hat{p})) & =2\left(\Sigma_{1} \Sigma_{j} x_{1, j} \log x_{1 j}+N \log N-\Sigma_{1} x_{1} \log x_{1} .\right. \\
& \left.-\Sigma_{j} x_{. j} \log x_{. j}\right)
\end{aligned}
$$

Cette expression suit asymptotiquement une loi de $\chi^{2}$ à $(r-1)$ (c - 1) degrés de liberté dans l'hypothèse d'indépendance. On remarquera son analogie formelle avec la valeur de $2 \mathrm{I}\left(\mathrm{p}^{*}: \mathrm{p}\right)$ dans le cas de la comparaison de $r$ échantillons.

\section{Excmple d'application:}

Pour l'expérimentation du débroussaillement sélectif (dégagements) des plantations résineuses, une application aérienne de 2.4.5-T a été pratiquée sur une parcelle de taillis enrésiné mettant en ceuvre $120 \mathrm{l} / \mathrm{ha}$ d'une émulsion à $3 \%$ d'un produit commercial à $710 \mathrm{~g} / 1$ d'acide 2.4.5-T: of. Arbonnter (1). Pour apprécier les résultats de ce traitement - et notamment la réduction du couvert - on a procédé sur les interbandes à un sondage systématique 
au $1 / 10$. Pour chaque brin de taillis d'une placette de sondage, on a ainsi relevé l'essence et une note de résistance (échelle conventionnelle de 0 à 6 ).

Les résultats d'ensemble du sondage sont reportés sur le tableau suivant:

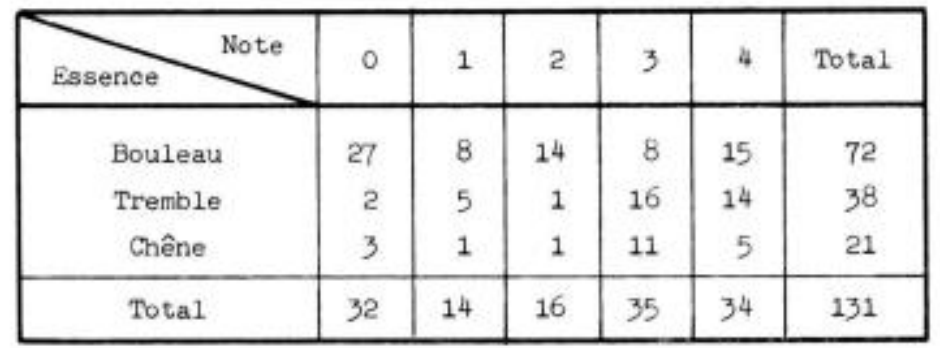

Tableau 2.2 - Sondages de contrôle - Mur de Sologne, parcelle E3

On a bien jei un tableau de contingence, chaque caractère étant siumultanément constaté sur les éléments de l'échantillon. Si les essences réagissent de façon comparable au produit, les caractères " ligne $n$ et "colonne " sont indépendants.

L'indépendance est testée en appliquant la formule (2, 2) cidessus, on a:

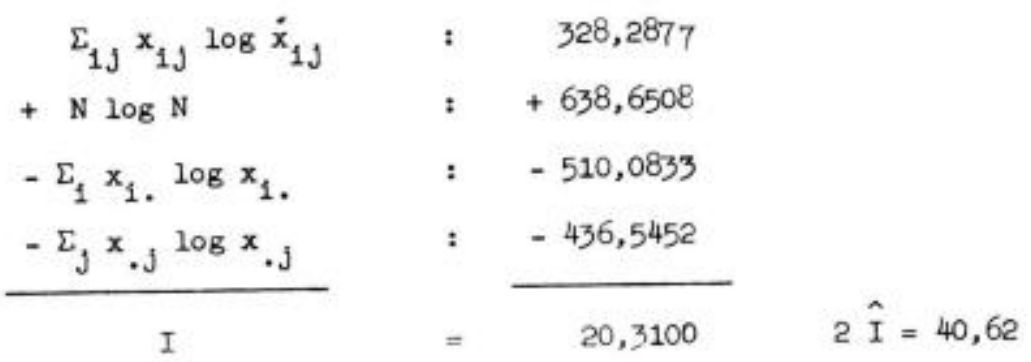

à $+\times 2-8$ degrés de liberté.

$$
\chi^{2}{ }^{2} .05=15,51 \quad \chi^{2}{ }^{2} 0.01=20,09
$$

La valeur trouvée pour 2 I est donc hautement significative. Cette conclusion n'a rien de surprenant: il est clair, lorsqu'on examine le tableau, que le comportement du houlean est à priori différent de celui du tremble et du chêne. 


\section{2 - Table de contingence ì triple entric.}

L'analyse de l'information offe de nombreuses possibilités dans le cas des tables de contingence à triple entrée et nous ne les traiterons pas toutes. La gamme étenduce des hypothèses que l'on pent tester résulte du fait que l'hypothèse nulle d'indépendance globale que l'on peut symboliquenent désigner par $\mathrm{H}_{2}(\mathrm{R} \times \mathrm{C} \times \mathrm{E})-\mathrm{F}$ désignant ici l'étage ou troisieme dimension de la table - peut être ¿léfinie comme l'intersection de diverses sous-hypothèses, successivement conditionnelles auxquelles correspond une décomposition additive de l'information. O 11 trouvera dans Kur.sack (8), chapitre 8 , une étude compléte des tables de contingence à triple entrée: dans ce domaine, l'analyse de l'information parait plus diversifiée que l'analyse classique qui utilise la loj de $\chi^{2}$.

\section{2, 21 - Indipendance globale.}

Un échantillon est constitué de $\mathrm{N}$ éléments sur lesquels on observe simultanément trois caractères correspondant à une classification par lignes (indexée par $i, 1 \leqslant i \leqslant r$ ), une classification par colonnes (indexée par $\mathrm{j}, 1 \leqslant \mathrm{j} \leqslant \mathrm{c}$ ) et une classification par étages (indexée par $k, 1 \leqslant k \leqslant d)$. Cne fréquence observée est désignée par $x_{\text {igh }}$ et les différentes fréquences marginales s'écrivent suivant la notation classique:

$$
\begin{aligned}
& \Sigma_{1} x_{1 j k}=x_{. j k} \quad \Sigma_{1} \Sigma_{j} x_{1 j k}=x_{. . k}=\Sigma_{j} x_{. j k} \\
& \Sigma_{1} x_{1 \ldots}=\Sigma_{j} x_{. j .}=\Sigma_{k} x_{. . k}=N
\end{aligned}
$$

L'hypothése d'indépendance des factems s'exprine, en indexant les probabilités de la mène maniere que les fréquences par:

$$
H_{2}: p_{1, k}=p_{1 . .} p_{. j}{ }^{p} \ldots k \quad p_{1 . .}>0, p_{, j}>0, p_{\ldots k}>0
$$

sous les conditions:

$$
\Sigma_{1} p_{1 \ldots}=\Sigma_{j} p_{. j .}=\Sigma_{k} p_{\ldots k}=1
$$

les sommations étant étendues de 1 i $r$ pour $i$, de 1 i c pour $j$, de 1 à d pour $\mathrm{k}$.

L.hypothèse alternative $H_{1}$ est définie par:

$$
\mathrm{H}_{1}: \mathrm{p}_{1, \mathrm{k}} \neq \mathrm{p}_{1 \ldots} \mathrm{p}_{. j} \mathrm{p}^{\mathrm{p} . \mathrm{k}}
$$


pour au moins un triplet (i, j, k) sous les seules conditions :

$$
p_{1 j k}>0 \quad \Sigma_{1 j k} p_{1 j k}=1
$$

On montre que l'on a toujours:

(2. 3)

$$
I\left(H_{1}: H_{2}\right)=N \Sigma_{1} \Sigma_{j} \Sigma_{k} p_{1 j k} \log \frac{p_{1 j k}}{p_{1 . .}{ }^{p} \cdot \jmath \cdot{ }^{p} . . k}
$$

$\mathrm{Si}(\mathrm{p})^{*}$ désigne la distribution conjuguée dont les paramètres sont ceux de la meilleure estimation sans hiais obtenue à partir de l'échantillon, on a pour l'estimation de l'information correspondante:

(2. 4)

$$
\hat{I}(p) *:(p)=\Sigma_{1 j k} x_{1 j k} \log \frac{x_{1 j k}}{N_{1} \ldots{ }^{p} \cdot J \cdot{ }^{p} . . k}
$$

Cette information est relative à des valeurs déterminées des probabilités marginales, done à une hypothèse $\mathrm{H}_{2}$ plus restrictive que celle que nous désirons vérifier. En désignant symboliquement par $\mathrm{H}_{2}(\mathrm{R})$ l'hypothèse spécifiant une valeur donnée $\mathrm{P}_{\text {... }}$ et de même $\mathrm{H}_{2}(\mathrm{C})$ et $\mathrm{H}_{2}(\mathrm{E})$, on peut considérer $\mathrm{H}_{2}$ comme les intersections d'hypothèses conditionnelles successives symbolisées par la formule:

$$
\mathrm{H}_{2}=\mathrm{H}_{2}(\mathrm{R}) \cap \mathrm{H}_{2}(\mathrm{C}) \cap \mathrm{H}_{2}(\mathrm{E}) \cap \mathrm{H}_{2}(\mathrm{R} \times \mathrm{C} \times \mathrm{E})
$$

L.hypothèse $\mathrm{H}_{2}(\mathrm{R} \times \mathrm{C} \times \mathrm{E})$ est l'hypothèse d'indépendance que nous voulons tester. L information $I\left[(p)^{*}:(p)\right]$ peut alors être décomposée additivement pour faire apparaitre le terme correspondant à l'hypothèse d'indépendance.

Les termes correspondants de la décomposition seront distriluces asymutotiquement comme $\chi^{2}$ avec respectivement $\mathrm{r}-1, \mathrm{c}-1$, $\mathrm{d}-1$ et $\mathrm{r} c \mathrm{~d}-1-(\mathrm{r}-1+\mathrm{c}-1+\mathrm{d}-1)$

$$
-(\mathrm{rcd}-\mathrm{r}-\mathrm{c}-\mathrm{d}+2) \text { degrés de liberté. }
$$


Le tableau d'analyse est le suivant:

\begin{tabular}{|c|c|c|}
\hline Nature de la composante & Information & Degres de liberté \\
\hline $\begin{array}{l}\text { Lipnes } H_{2}(R) \\
\beta_{1} . .-x_{1} \ldots / N\end{array}$ & $2 \Sigma_{1} x_{1 . .} \log \frac{x_{1} \ldots}{N_{1} \ldots}$ & $r-1$ \\
\hline $\begin{array}{l}\text { Colonnes } \mathrm{H}_{2}(\mathrm{C}) \\
\mathrm{s}_{. j .}=\mathrm{x}_{.3 .} \text { N }\end{array}$ & 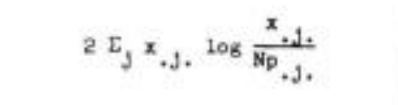 & c -1 \\
\hline $\begin{array}{l}\text { Etages } \mathrm{H}_{2}(\mathrm{E}) \\
\mathrm{B}_{\ldots \mathrm{k}}-\mathrm{x} \ldots \mathrm{K}^{\prime N}\end{array}$ & $2 I_{k} x \ldots k \log \frac{x_{\ldots k}}{k_{p_{i k k}}}$ & $d-1$ \\
\hline $\begin{array}{l}\text { Indegendarioe } \\
\mathrm{H}_{2}(\mathrm{~A} \times \mathrm{C} \times \mathrm{B})\end{array}$ & $2 \Sigma_{1 j k} x_{1, j k} \log \frac{\mathrm{N}^{2} x_{1 j k}}{x_{1 . n^{x} \cdot J \cdot{ }^{x} \ldots k}}$ & red $=r-c-d+2$ \\
\hline $2 \hat{I}[(p) *:(p))$ & $2 \Sigma_{1, j k} x_{1 j k} \log \frac{x_{1, j k}}{\operatorname{Np}_{1 . . P^{p} . j . P^{p} \ldots k}}$ & $\mathrm{red}-1$ \\
\hline
\end{tabular}

Tableat 2.3 - Indépendance; table à triple entréc.

$$
\text { ou } \Sigma_{i j k} \text { signifie } \underset{i=1}{\mathrm{r}} \underset{j=1}{\mathrm{z}} \stackrel{\mathrm{d}}{\mathbf{\Sigma}}
$$

On testera l'indépendance en comparant aux valeurs de $\chi^{2}$ correspondant au nombre de degrés de liberté indiqué et au seuil de probabilité choisi, l'expression figurant sur l'avant-dernière ligne de ce tableau qui peut encore s'écrire:

$$
\begin{aligned}
2 \hat{I} & =2\left(\Sigma_{1 j k} x_{1 j k} \log x_{1, j k}+2 N \log N-\Sigma_{1} x_{1 . .} \log x_{1} \ldots\right. \\
& \left.-\Sigma_{j} x_{. j .} \log x_{. j .}-\Sigma_{k} x_{\ldots k} \log x_{\ldots k}\right)
\end{aligned}
$$

\section{22 - Indépendance d'une classification par rapport aur.} autres.

On peut aussi se demander si liune des classifications de la table est indépendante des deux nutres.

Lhypothèse $\mathrm{H}_{2}$ est alor's exprimée par la relation:

$$
\begin{aligned}
& H_{2}: p_{1 j k}=p_{\ldots k} \cdot p_{1 j} . \quad p_{\ldots k}>0 \quad p_{1 j .}>0 \\
& \text { avec } \sum_{k} p_{\ldots k}=1 \quad k=1,2 \ldots, d \Sigma_{1 j} p_{1 j}=1
\end{aligned}
$$


alors que l'hypothèse $\mathrm{H}_{1}$ est:

$$
p_{1 j k} \neq p_{\ldots k} \cdot p_{1 j} .
$$

pour au moins un triplet $(\mathrm{i}, \mathrm{j}, \mathrm{k})$

$$
p_{1 j k}>0 \quad \Sigma_{1} \Sigma_{j} \Sigma_{k} p_{1 j k}=1
$$

Si l'hypothèse $\mathrm{H}_{2}$ est vérifiée, on peut écrire en utilisant la relation (2, 6):

$$
\begin{aligned}
& p_{. j k}=\Sigma_{1} p_{1 \downarrow k}=p_{\ldots k} \Sigma_{1} p_{1 j .}=p_{\ldots k} \cdot p_{. j} . \\
& p_{1, k}=\sum_{j} p_{1, k}=p_{\ldots k} \Sigma_{j} p_{1 j .}=p_{\ldots k} \cdot p_{1} .
\end{aligned}
$$

ce qui montre que l'indépendance de l'étage par rapport aux lignes et aux colonnes entraine l'indépendance des lignes et des colonnes entre elles.

Il y a deux manières d'obtenir la composante d'information permettant le test de l'hypothèse $\mathrm{H}_{2}$. La première consiste à estimer $2 \mathrm{I}\left[(\mathrm{p})^{3}:(\mathrm{p})\right]$ et à la décomposer, comme on l'a déjà fait chaque fois auparavant, en trois termes: le premier concernant le test des p..k par rapport aux p..k, le second un test analogue sur p.is et le dernier s'appliquant à l'hypothèse générale d'indépendance. Ces trois termes sont additifs et correspondent à la suite d'hypothèses:

$$
\mathrm{H}_{2}=\mathrm{H}_{2}(\mathrm{E}) \cap \mathrm{H}_{2}(\mathrm{RC}) \cap \mathrm{H}_{2}(\mathrm{E} \times \mathrm{RC})
$$

La seconde méthode consiste à décomposer l'hypothèse d'indépendance des trois classifications en une hypothèse d'indépendance de $\mathrm{R}$ par rapport à $\mathrm{C}$ associée à celle de $\mathrm{E}$ par rapport à $\mathrm{RC}$, soit symboliquement :

$$
\mathrm{H}_{2}(\mathrm{R} \times \mathrm{C} \times \mathrm{E})=\mathrm{H}_{2}(\mathrm{R} \times \mathrm{C}) \cap \mathrm{H}_{2}(\mathrm{E} \times \mathrm{RC})
$$


On a alors le tableau suivant d'analyse de l'information:

\begin{tabular}{|c|c|c|}
\hline Nature de la eonposante & Information & Degrés de liberte \\
\hline $\begin{array}{c}\text { Lignes } x \text { colosures } \\
H_{2}(\mathrm{H} \times \mathrm{C})\end{array}$ & $2 \Sigma_{1 j} x_{1 j} . \log \frac{x_{1 j}}{x_{1 . n^{x}+3}}$ & $(r-1)(0-1)$ \\
\hline 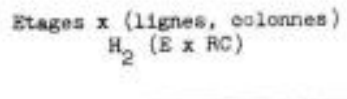 & $2 E_{1, j k} x_{1 / k} \log \frac{N x_{1, j k}}{x_{1 j .} x_{\cdots k}}$ & $(r e-1)$ \\
\hline $\begin{array}{l}\text { Indépendance } \\
\mathrm{H}_{2}(\mathrm{~A} \times \mathrm{C} \times \mathrm{B})\end{array}$ & $2 \Sigma_{1 j k} x_{1 j k} \log \frac{x^{2} x_{1 j k}}{x_{1 . n^{x} \cdot j \cdot 0^{x} \ldots k}}$ & red $-x-e-d+2$ \\
\hline
\end{tabular}

Tableau 2.4 - Indépendance (étages $\mathrm{x}$ lignes, oulonnes)

On testera par $\chi^{2}$ l'expression figurant sur la seconde ligne du tableau dont le développement est explicité dans l'exemple qui suit.

\section{Exemple d'application:}

Le tableau (2.5) rassemble les résultats des sondages réalisés pour

\begin{tabular}{|c|c|c|c|c|c|c|c|c|}
\hline Dose & Basence & 0 & 1 & 2 & 3 & 4 & 5 & Total \\
\hline \multirow{5}{*}{$3.5 * \mathrm{P} 80$} & Bouleau & 228 & 143 & 162 & 180 & 23 & 0 & 736 \\
\hline & Trenble & 50 & 51 & 97 & 188 & 73 & 0 & 459 \\
\hline & Cnâta1gnier & 13 & 18 & 19 & 57 & 175 & 0 & 282 \\
\hline & Chêne & 0 & 0 & 4 & 9 & 10 & 0 & 23 \\
\hline & Total & 291 & 212 & 282 & 434 & 281 & 0 & 1500 \\
\hline \multirow{5}{*}{$3 x P 80$} & Bouleau & 195 & 62 & 51 & 67 & 14 & 0 & 389 \\
\hline & Tremble & 22 & 33 & 56 & 85 & 68 & 0 & 264 \\
\hline & Cnâtalgnier & 3 & 1 & 0 & 9 & 30 & 9 & 52 \\
\hline & Chêne & 4 & 5 & 10 & 37 & 17 & 0 & 73 \\
\hline & Total & 224 & 101 & 117 & 198 & 129 & 9 & 778 \\
\hline \multirow[t]{4}{*}{ Ensenble } & Bouleau & 423 & 205 & 213 & 247 & 37 & 0 & 1125 \\
\hline & Tremble & 72 & 84 & 153 & 273 & 141 & 0 & 723 \\
\hline & Châta1gnier & 16 & 19 & 19 & 66 & 205 & 9 & 334 \\
\hline & Chêne & 4 & 5 & 14 & 46 & 27 & 0 & 96 \\
\hline Total & Général & 515 & 313 & 399 & 632 & 410 & 9 & 2278 \\
\hline
\end{tabular}

Tableat 2.5 - Traitement hélicoptẻre Mur de Sologne - Résultats globaux (*)

(*) On peut s'interroger sur la nécessité du maintien de la classe 5. Cependant, la différence entre les notes 4 et 5 , dans l'échelle adoptée, est très nette; d'autre part, pour le traitement $3 \%$, la proportion de chátaigniers observée $(17,3 \%)$ est trop importante pour qu’on puisse la négliger. 
contròler les résultats de lapplication aérienne dont il a déjà été question au paragraphe 2. 21 ci-avant. Sur deux groupes de parcelles, deux traitements ont été mis en ceuvre, correspondant à des concentrations différentes $(3 \%$ et $3,5 \%$ ) du produit à base de 2.4.5-T. On est en droit de se demander $3 i$ les différentes essences ont réagi de façon identique à une modification de la concentration du produit. S'il en était ainsi, la classification " dose " serait indépendante des classifications par essences et par symptómes de résistance (notes de 0 à 6 ).

L'information relative à l'hypothèse d'indépendance $\mathrm{H}_{2}(\mathrm{E} \times \mathrm{RC})$, $\mathrm{E}$ désignant la concentration, s'écrit en développant :

$$
\begin{aligned}
& \hat{I}=\Sigma_{1, j, k} x_{1 j k} \log x_{1 j k}+N \log N-\Sigma_{1, j} x_{1 j .} \log x_{1, j} . \\
& \quad-\Sigma_{k} x_{\ldots k} \log x_{\ldots k} \\
& \text { Ici, } r-4 c-6 d-2 \\
& \quad x_{. .1}-1500 x_{. .2}-778 \quad N=2278
\end{aligned}
$$

les valeurs $\mathrm{x}_{11}$. figurent sous la table, au tableau " ensemble ".

On trouve:

$$
\begin{gathered}
\mathrm{I}=10480,026+17611,357-11823,963-16148,783 \\
=118,637 \\
2 \mathrm{I}=237,27
\end{gathered}
$$

Avec 23 degrés de liberté $\chi_{0,05}^{2}=35,17 \quad \chi_{0,01}^{2}=41,64$ l'indépendance n'est donc pas vérifiée. Le comportement du bouleau parait à première vue responsable de ce résultat: le pourcentage de 0 (arbres morts) qui est d'environ $50 \%$ à la concentration de $3 \%$ n'est plus que de $31 \%$ à la concentration de $3,5 \%$. Par contre, il est plus difficile de porter un jugement sur le comportement respectif des autres essences.

\section{Remarque:}

L'hypothèse d'indépendance $\mathrm{H}_{2}(\mathrm{E} \times \mathrm{RC})$ exprimée par (2.6) (2. 6)

$$
p_{i j k}=p_{. . k} \cdot p_{i j .}
$$


est équivalente à $\left(^{*}\right)$ :

$$
p_{1, k}=p_{. . k} p_{1 . .} \text { et } p_{i j k}=p_{i j} . p_{1, k} / p_{1 . .}
$$

soit

$$
\begin{array}{ll}
\mathrm{H}_{2}(\mathrm{R} \times \mathrm{E}) & \text { et } \mathrm{H}_{2}(\mathrm{C} / \mathrm{R} \times \mathrm{E} / \mathrm{R}) \\
\mathrm{H}_{2}(\mathrm{~B} \times \mathrm{RC})=\mathrm{H}_{2}(\mathrm{C} / \mathrm{R} \times \mathrm{E} / \mathrm{R}) \cap \mathrm{H}_{2}(\mathrm{R} \times \mathrm{E})
\end{array}
$$

La classification "étages " est indépendante de la classification a lignes " et "colonnes $n$ si les classifications " lignes n et " colonnes n sont indépenclantes et que les classifications "colonnes " et " étages " sont conditionnellement indépendantes, étant donné la classification par "lignes ".

Du point de vue de l'information, on obtient une nouvelle décomposition additive permettant la vérification des sous-hypothèses pré-

\begin{tabular}{|c|c|c|}
\hline Composantes & Information & Degrús de 11berté \\
\hline $\begin{array}{l}\text { 11enes } \times \text { étages } \\
\text { (A } \times \text { B) }\end{array}$ & $2 \sum_{1 k} x_{1, k} \log \frac{N x_{1, k}}{x_{1 \ldots} x_{\ldots k}}$ & $(r-1)(d-1)$ \\
\hline $\begin{array}{c}\text { (Colonnes, 11gnes) } \\
\times \text { (etages, 11gnes }) \\
\mathrm{H}_{2}(\mathrm{C} / \mathrm{A} \times \mathrm{E} / \mathrm{R})\end{array}$ & $2 E_{1 j k} x_{1 j k} \log \frac{x_{1, k}}{\frac{x_{1 j} x_{1, k}}{x_{1 \ldots}}}$ & $r(c-1)(d-1)$ \\
\hline $\begin{array}{c}\text { Etage } x \text { (11gnes oolonnes) } \\
\mathrm{H}_{2} \quad(\mathrm{E} \times \mathrm{RC})\end{array}$ & $2 \Sigma_{1, k} \log \frac{N x_{1, k}}{x_{1 J} x_{\ldots k}}$ & $(\mathrm{~d}-1) \quad(\mathrm{re}-1)$ \\
\hline
\end{tabular}
cédentes par le $\chi^{2}$ asymptotique.

Les expressions de l'information sont respectivement:

Ces expressions se développent encore en facteurs de la forme $\mathrm{n} \log \mathrm{n}$.

On aurait pu écrire de même:

$$
\mathrm{H}_{2}(\mathrm{~B} \times \mathrm{RC})=\mathrm{H}_{2}(\mathrm{R} / \mathrm{C} \times \mathrm{E} / \mathrm{C}) \text { in } \mathrm{H}_{2}(\mathrm{R} \times \mathrm{C})
$$

et obtenir une analyse de l'information permettant de tester les hypothèses du second membre.

(*) Faire la sommation de $p_{14 k}$ pour $j=1$ à 6 
2. 23 - Comparaison de $r$ tables de contingence à double entrée.

Nous avons vu précédenment (paragraphe 1. 2) qu'il était possible de comparer entre eux $r$ échantillons d'une distribution multinomiale à c classes et notamment d'en tester l'homogénéité globale. On peut étendre le test d'homogénéité aux tables de contingence à double entrée $(r \times c)$.

L'analyse de l'information s'inspire de l'analyse d'une table de contingence à triple entrée mais les restrictions doivent en ètre modifiées.

L'hypothèse alternative est:

$$
\begin{aligned}
& \mathrm{H}_{1}: \mathrm{p}_{1 j \mathrm{k}} \neq \mathrm{p}_{1 \mathrm{j} .} \quad \begin{array}{l}
1=1,2 \ldots \mathrm{r} \quad \mathrm{j}=1,2 \ldots \mathrm{c} \\
\mathrm{K}=1,2 \ldots \mathrm{d}
\end{array} \\
& \Sigma_{1} \Sigma_{j} \mathrm{p}_{1 j \mathrm{k}}=1
\end{aligned}
$$

L'hypothèse nulle $\mathrm{H}_{2}$ est au contraire:

$$
H_{2}: p_{1, j k}=p_{1 j} . \forall 1, j \quad \Sigma_{1} \Sigma_{j} p_{1 j .}=1
$$

La meilleure estimation de $\mathrm{P}_{11}$. est:

$$
\hat{\mathrm{p}}_{1 \mathrm{~J} \cdot}=\mathrm{x}_{1 \mathrm{~J} \cdot} \cdot \mathrm{N}
$$

et linformation $\hat{\mathrm{I}}\left[(\mathrm{p})^{*}:(\mathrm{p})\right]$ se décompose encore additivement

\begin{tabular}{|c|c|c|}
\hline Composante & Information & Degrés de l1berté \\
\hline 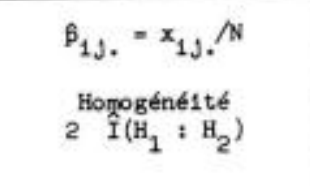 & $\begin{array}{l}2 \Sigma_{1 \jmath} x_{1 J .} \log \frac{x_{1 j}}{N p_{1 j}} \\
2 \Sigma_{1 j k} \log \frac{N x_{1 j k}}{x_{1 j .}{ }^{x} \ldots k}\end{array}$ & $(d-1)\left(r_{c}-1\right)$ \\
\hline Total $2 \hat{I}((p) *:(p))$ & $2 \Sigma_{1, k} x_{1 j k} \log \frac{x_{1 j k}}{x_{\ldots k} p_{1 j}}$ & $r(e d-1)$ \\
\hline
\end{tabular}
selon la formule:

$$
\hat{I}((p) *(p))=\hat{I}((\hat{p}):(p))+\hat{I}((p) *:(\hat{p}))
$$

pour donner le tableau d'analyse suivant:

Tableau 2.6 - Homogênéité de d échantillons $\mathrm{r} \times \mathrm{c}$ 
La composante d'homogénéité est, sots 1'hypothèse nulle, asymptotiquement distribuée comme $\chi^{2}$ avec $(d-1)$ (rc - 1) degrés de liberté et on la testera au senil de probabilité choisi. Elle s'écrit encore :

(2. 10)

$$
\begin{aligned}
2 \hat{I} & =2\left(\Sigma_{1, j k} \log x_{i, j k}+N \log N-\Sigma_{i j} x_{1, j .} \log x_{i j .} .\right. \\
& \left.-\Sigma_{k} x_{\ldots k} \log x_{\ldots k}\right)
\end{aligned}
$$

\section{Fixemple d'application:}

Le traitement aérien à $3 \%$ P\$O a été appliqué sur 3 parcelles différentes et contiguës qui ont fait l'objet de sondages distincts dont les résultats sont reportés sur le tableatı 2.7. On désirerait connaitre si l'application a été honogène sur l'ensemble des parcelles traitées. Si l'on considère chaque relevé de parcelle comme un échantillon, l'analyse de f'information permet de contrỏler l'hypothèse d'homogénéité, L'indice i étant attribué à l'essence, l'indice $j$ à la note de résistance et l'indice $k$ ì la parcelle. On a :

$$
\begin{array}{lll}
\mathrm{r}=4 & \mathrm{c}=6 \quad \mathrm{~d}-3
\end{array}
$$

\begin{tabular}{|c|c|c|c|c|c|c|c|c|}
\hline Parcelle & Essence & 0 & 1 & 2 & 3 & 4 & 5 & Total \\
\hline \multirow{5}{*}{ C3 } & Bouleau & 84 & 21 & 15 & 22 & 4 & 0 & 146 \\
\hline & Tremble & 1 & 9 & 15 & 18 & 7 & 0 & 50 \\
\hline & Châtaignier & 3 & 0 & 0 & 1 & 2 & 0 & 6 \\
\hline & Chêne & 0 & 2 & 5 & 24 & 6 & 0 & 37 \\
\hline & Total & 88 & 32 & 35 & 65 & 19 & 0 & 239 \\
\hline \multirow{5}{*}{03} & Bouleau & 73 & 24 & 15 & 17 & 0 & 0 & 129 \\
\hline & Treable & 19 & 20 & 29 & 33 & 3 & 0 & 104 \\
\hline & Châtafinter & 0 & 1 & 0 & 4 & 8 & 6 & 19 \\
\hline & Chêne & 4 & 3 & 5 & 9 & 10 & 0 & 31 \\
\hline & Total & $90^{\circ}$ & 48 & 49 & 63 & 21 & 6 & 283 \\
\hline \multirow{5}{*}{$\mathrm{CH}_{4}$} & Bouleau & 38 & 17 & 21 & 28 & 10 & 0 & 114 \\
\hline & Tremble & 2 & 4 & 12 & 34 & 58 & 0 & 110 \\
\hline & Châtaígnier & 0 & 0 & 0 & 4 & 20 & 3 & 27 \\
\hline & Chêne & 0 & 0 & 0 & 4 & 1 & 0 & 5 \\
\hline & Total & 40 & 21 & 33 & 70 & 89 & 3 & 256 \\
\hline
\end{tabular}

Traitement aérien, Mur de Solngne - Relevé des parcelles traitées à 3 \%. 


\begin{tabular}{|c|r|r|r|r|r|r|c|}
\hline & 0 & 1 & 2 & 3 & 4 & 5 & Total \\
\hline Bouleau & 195 & 62 & 51 & 67 & 14 & 0 & 389 \\
Tremble & 22 & 33 & 56 & 85 & 68 & 0 & 264 \\
Châtalgnier & 3 & 1 & 0 & 9 & 30 & 9 & 52 \\
Chêne & 4 & $\frac{5}{10}$ & $\frac{10}{117}$ & $\frac{37}{198}$ & $\frac{17}{129}$ & $\frac{0}{9}$ & $\frac{73}{778}$ \\
\hline Total & 224 & 101 & 117 & \\
\hline
\end{tabular}

Tableau 2.7 - Totaux, ensemble des parcelles $=\mathrm{x}_{\mathrm{i}}$.

Les valeurs de $x_{, \text {, }}$ sont respectivement $239,283,256$. Les valeurs de $\mathrm{x}_{11}$. sont celles du tableau séparé et $\mathrm{N}=778$.

On trouve:

$$
\begin{array}{lcc}
\Sigma_{1 j k} x_{1 j k} \log x_{i j k} & 2515,6580 & \begin{array}{l}
\text { Nombre de degrés } \\
\text { de } 11 \text { berté }
\end{array} \\
+\mathrm{N} \log N & +5178,933 e & (\mathrm{~d}-1)(\mathrm{rc}-1)=2 \times 19 \\
-\Sigma_{\mathrm{k}} \mathrm{x}_{\ldots k} \log \mathrm{x}_{\ldots \mathrm{k}} & -4326,1017 & \mathrm{~d} 1=38 \\
-\Sigma_{1 j} \mathrm{x}_{1 j .} \log \mathrm{x}_{1 \mathrm{j} \cdot \mathrm{n}} & -3239,9249 &
\end{array}
$$

Avec 40 degrés de liberté $\chi^{2}{ }_{0,05}=55,76$ et $\chi^{2}{ }_{0,01}=63,69$. A fortiori, la valeur trouvé $2 \hat{\mathrm{I}}-257.12$ est hattement significative : les échantillons ne sont pas homogènes et l'on peut conclure que l'application a donné, pour chaque parcelle, des effets qui ne peuvent être considérés comme identiques.

\section{Resuarque:}

On notera l'identité des expressions et des degrés de liberté de la distribution asymptotique de $\chi^{2}$ pour les informations permettant de tester respectivement:

- l'indépendance d'une classification par rapport aux deux autres,

- l'homogénéité de plusicurs échantillons de tables à double entrée.

En fait, les problèmes sont tout à fait différents et il en va de même des hypothèses: dans le cas de l'indépendance, les probabi- 
lités a d'étage " ne sont pas identiques dans l'hypothèse nulle: on peut seulement admettre leur existence. Dans la comparaison de c échantillons, ce sont les rc probabilités pus. que l'on peut considérer comme identiques dans chacun des échantillons soumis au test lorsque celui-ci n'est pas significatif.

Formellement, les hypotheses nulles se traduisent par les formules suivantes:

$$
p_{1, j k}=p_{1, J .} \cdot p_{\ldots k} \quad \forall 1, j, k
$$

pour l'indépendance étages $\mathrm{x}$ (lignes, colonnes)

$$
p_{1, k}=p_{1 j} . \forall_{1, j}
$$

pour l'homogénéité de $r$ tables (lignes, colonnes).

2. 24 - Décomposition de la composante d'homogénéité.

Lat composante d'homogénéité $(\mathrm{R} \times \mathrm{C}$ ) peut encore se décomposer en deux facteurs additifs correspondant au fait que l'hypothèse d'homogénéité $\mathrm{H}_{2}\left(\mathrm{R} \times \mathrm{C}^{\prime}\right)$ peut ètre consitlérée conme l'intersection d'une hypothèse d'homogénéité en C et d'une hypothèse d'homogénéité conditionnelle en D liée par C.

$$
\mathrm{H}_{2}(\mathrm{C} \times \mathrm{D})=\mathrm{H}_{2}(\mathrm{C}) \cap \mathrm{H}_{2}(\mathrm{D} / \mathrm{C})
$$

On peut tester chacun des membres de droite de cette expression par deux estimations de l'information comme on la fait an paragraphe 2. 22 dans la remarque qui termine ce paragraphe.

\section{25 - Homogénéité conditionnelle.}

11 est encore possible, dans le cas de r échantillons de tables de contingence à double entrée de tester, par exemple pour une ligne i. l'hypothèse de l'homogénéité des échantillons relativement à la classification en colonnes. 
L'hypothèse nulle $\mathrm{H}_{2}$ est alors exprimée par:

(2. 11)

$$
\begin{gathered}
H_{2}: p_{1 j k}=p_{1 j .} \quad 1 \text { donné, } \quad \begin{array}{l}
J=1,2 \ldots . c \\
K=1,2 \ldots . d
\end{array} \\
\sum_{1 j .}=p_{1 .} .
\end{gathered}
$$

l'hypothèse alternative étant

$$
H_{1}: \quad p_{1 j k} \neq p_{1 j} . \quad \sum_{k} p_{1 j k}=p_{1 j} .
$$

\begin{tabular}{|c|c|c|}
\hline Composante & Information & Degrás de ilberté \\
\hline 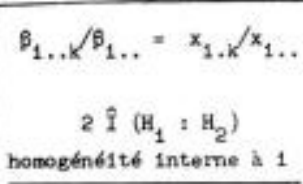 & 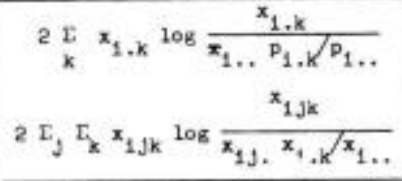 & $(c-1)(d-1)$ \\
\hline $2 \hat{\mathrm{I}}((p) *(p))$ & $2 \Sigma_{j} \Sigma_{k} x_{1 j k} \log \frac{x_{1 j k}}{x_{1 j} \cdot p_{1, k} p_{1} \ldots}$ & $e\langle d-1\rangle$ \\
\hline
\end{tabular}

Le tableat d'analvse de l'information est alors le suivant:

Tableau 2.8 - Homogénéité conditionnelle (ligne i)

L'expression de l'information permettant le test de l'homogénéité des $\mathrm{r}$ échantillons de la ligne $\mathrm{i}$ pour la classification $\mathrm{j}$, s'écrit:

$$
\begin{aligned}
2 \hat{I}= & 2\left(\Sigma_{j k} x_{1 j k} \log x_{1 j k}+x_{1 . .} \log x_{1 . .}-\Sigma_{j} x_{1 j .} \log x_{1 j} .\right. \\
& \left.-\Sigma_{k} x_{1 . k} \log x_{1, k}\right)
\end{aligned}
$$

elle tend asymptotiquement vers une clistribution de $\chi^{2}$ à $(c-1)$ (d-1) degrés de liberté.

\section{Exemple d'application:}

Supposons que nous vetillons contróler si le bouleau a réagi de façon identique au traitement à $3 \%$ dans les parcelles C3, G3 et 
G4 (tableau 2.7 ci-avant). On testera l'homogénéité des 3 échantillons constitués par la ligne "bouleau " $(\mathrm{i}=1)$ par rapport à la classification " colonnes " ( 0 à 5 ).

$$
\begin{aligned}
& \text { Ici } \mathrm{x}_{1 . .}=389 \\
& \mathrm{x}_{1,1}=239 \quad \mathrm{x}_{1,2}=283 \quad \mathrm{x}_{1,3}=256
\end{aligned}
$$

Les $\mathrm{x}_{1 \mathrm{j}}$. sont les chiffres de la ligne "bouleau " des totaux de l'ensemble des parcelles: $195,62, \ldots, 14,0$.

On a alors:

$$
\begin{array}{lll}
\Sigma_{j k} \cdot x_{1 j k} \log x_{1 j k} & 1395,2092 & \text { d.1. }(c-1)(d-1) \\
+x_{1} . . \log x_{1} . . & +2319,8324 & =5 \times 2=10 \\
-\Sigma_{j} x_{1 j} \cdot \log x_{1 j} & -1894,4490 & x_{0.05}^{2}=18,31 \\
\frac{-\Sigma_{k} x_{1 j k} \log x_{1 j k}}{\hat{I}}=\frac{-1803,3016}{17,2910} & x_{0.01}^{2}=23,21 \\
& 2 \hat{I}=34,58
\end{array}
$$

La valeur trouvée pour 2 I est très significative. II y a done, dans chaque parcelle traitée, des différences de comportenent du bouleau qui sont sans doute dues à un manque de régularité de l'application, les différences de station étant peu prononcées. 


\section{III - CONCLUSIONS}

Nous avons voulu mettre en lumière les possibilités offertes par lanalyse de l'information pour tester un certain nombre d'hypothèses lorsquion se trouve confronté avec des distributions multinomiales. Le chercheur se trouvera souvent dans cette situation, notamment lorsque les caractères olsservés sont de nature qualitative et ne peuvent être exprinés par une ou plusieurs grandeurs mesurables. En matière forestière, cette difficulté se rencontre dans de nombretuses disciplines, chaque fois quil s'agit de relever des caractères phénologiques ou floristiques: présocité du déhourrement. caractéristiques morphologiques din plant, réaction d'un arbre ou d'une souche à l'application de produits chimiques herbicides, etc... Alors l'expérimentateur se sent désarmé et tente de se raccrocher a l'analve de variance en utilisant des expédients: il essaie stucessivenent de multiples transformations qui, parfois, s'avèrent impuissantes et presque toujours l'embarrassent lorsqu'il doit conclure.

Il serait abusif de prétendre que l'analyse de l'information constitue toujours la seule méthode à larquelle on puisse se confier dande telles circonstances. I a méthode dit test $\chi^{2}$ est trop connue pour qu'il soit nécessaire d'en rappeler l'existence: elle peut être appliquée aut test d'homogénéité d'un échantillon d'une loi binomiale ou d'indépendance d'une table de contingence à double entrée - voir. par exemple, Sxedecok (10), chap. 9 et 16. Cependant, lanalyse de l'information nous parait beaucoup plus féconde et plus commode d'emploi.

Sur le plan des calculs, lanalyse de l'information se présente en effet comme une stite d'additions lorsqu'on dispose d'une table de 11 log $n$. Lorsque les valeurs de $n$ sont grandes, ou que le nombre d'échantillons ou de classes est élevé. il est indiqué d'utiliser le calcul automatique: la plupart des calculatrices électroniques englobent la function $\log _{e}$ dans les fonctions standard et la programmation est très simple, surtout lorsqu'on utilise un langage symbolique comme 1'Algol. Les méthodes que nous avons exposées seront toutes programmées par la Station de Biométrie et, dès lors, les calculs ne poseront aucun problème. 
La décomposition des quantités d'information en termes additifs correspondant à un certain nombre de sous-hypothèses dont l'intersection constitue l'hypothèse à laquelle est attachée la quantité d'information globale est une méthode extrémement élégante et, de surcroit, efficace. Elle n'est pas sans analogie avec l'analyse de variance et il n'y a là rien de surprenant puisque, dans l'analyse de variance, les sommes des carrés ne sont pas autre chose que des $\gamma^{2}$.

Au demeurant, nous nous sommes volontairement limités, dans l'exposé des applications pratiques étayé par des exemples, au cas de la distribution multinomiale. Des développements comparables sont possibles pour la distribution de Poisson et surtout pour la distribution multinormale: ils conduisent dans ce cas à de nombreux tests d'un grand intérêt.

En définitive, l'analyse de l'information constitue une vaste synthèse qui permet d'approcher par des voies nouvelles et de retrouver la plupart des méthodes les plus connues de l'analyse statistique. Elle ne pouvait rester totalement inconnue des chercheurs qui, aujourd'hui, ont un besoin de plus en plus pressant de cet outil efficace. 


\section{BIBLIOGRAPHIE}

1. Arbonnier (P.). - Premiers essais de traitement par hélicoptère pour le dégagement sélectif de plantations résineuses. Communication au $3^{\circ}$ Congrès International de PAviation Agricole, 1966.

2. Burghausex (R.). - Statistiche Auswertung von Vegetationsbeobachtungen in Feldversuchen. Internationale Tagung über mathematische Statistik und ihre Anwendungen, Berlin, 1964, pp. 43-48.

3. DAgNele (P.). - A propos des transformations de variables. BiométricPraximétrie, t. IV, $n^{\circ} 2,1965$, pp. 59-78.

4. Dufresoy (J.). - Quelques méthodes statistiques utilisables en agronomie. Cahier des Ingénieurs Agronomes, $\mathrm{n}^{\circ}$ 194, 1965, pp, 35-39.

5. Fisher (R.A.) and Yatrs (F.), - Statistical tables for biological agricultural and medical research. Oliver and Boyd, Edinbourg, 1953.

6. JerFers (J.N.R.). - Experimental design and analysis in Forest Research, Chap. 3. Almqvist et Wiksell, Stockholm, 1959.

7. Krndall (M.G.) and StunRT (A.). - The advanced theory of Statistics, 3 vol. Charles Griffin et $\mathrm{C}^{\circ}$, Londres, 1958 .

8. Kullzack (S.). - Information theory and statistics, 395 p, John Wiley and Sons, New-York, 1959.

9. Rives (M.), - Sur l'analyse de la variance. I. Emploi de transformations. Annales de l'Institut National de la Recherche Agronomique, Série B, $10-3,1960$.

10. SNenecor (G.W). - Statistical methods, $5^{\circ}$ ed., 534 p. The Towa University Press, Ames, 1956.

11. Tortrat (A.). - Calcul des probabilités, 168 p. Masson et Cie, Paris, 1963.

12. Vessereav (A.). - Méthodes statistiques en biologie et en agronomie, $2^{*}$ éd., 538 p., J.B. Baillière et fils, Paris, 1960.

13. Yaglom (A.M.) et Yaglom (J.M.). - Probabilité et information, 175 p. Dunod, Paris, 1959. 
TABLE DES VALEURS DE $\mathrm{n} \log \mathrm{n}$

$\begin{array}{rrrrrrrrrr}N & N * L N(N) & N & N \because L N(N) & N & N \because L N(N) & N & N * L N(N) & N & N * L N(N) \\ 1 & .0000 & 21 & 63.9350 & 41 & 152.2565 & 61 & 250.7633 & 81 & 355.9504 \\ 2 & 1.3863 & 22 & 68.0029 & 42 & 156.9821 & 62 & 255.8823 & 82 & 361.3510 \\ 3 & 3.2958 & 23 & 72.1164 & 43 & 161.7316 & 63 & 261.0175 & 83 & 366.7638 \\ 4 & 5.5452 & 24 & 76.2733 & 44 & 166.5043 & 64 & 266.1685 & 84 & 372.1886 \\ 5 & 8.0472 & 25 & 80.4719 & 45 & 171.2998 & 65 & 271.3352 & 85 & 377.6254 \\ 6 & 10.7506 & 26 & 84.7105 & 46 & 176.1175 & 66 & 276.5172 & 86 & 383.0739 \\ 7 & 13.6214 & 27 & 88.9876 & 47 & 180.9569 & 67 & 281.7144 & 87 & 388.5340 \\ 8 & 16.6355 & 28 & 93.3017 & 48 & 185.8176 & 68 & 286.9265 & 88 & 394.0056 \\ 9 & 19.7750 & 29 & 97.6516 & 49 & 190.6992 & 69 & 292.1533 & 89 & 399.4886 \\ 10 & 23.0259 & 30 & 102.0359 & 50 & 195.6012 & 70 & 297.3947 & 90 & 404.9829 \\ 11 & 26.3768 & 31 & 106.4536 & 51 & 200.5231 & 71 & 302.6503 & 91 & 410.4882 \\ 12 & 29.8189 & 32 & 110.9035 & 52 & 205.4647 & 72 & 307.9200 & 92 & 416.0046 \\ 13 & 33.3443 & 33 & 115.3847 & 53 & 210.4255 & 73 & 313.2035 & 93 & 421.5318 \\ 14 & 36.9468 & 34 & 119.8963 & 54 & 215.4051 & 74 & 318.5008 & 94 & 427.0697 \\ 15 & 40.6208 & 35 & 124.4372 & 55 & 220.4033 & 75 & 323.8116 & 95 & 432.6183 \\ 16 & 44.3614 & 36 & 129.0067 & 56 & 225.4197 & 76 & 329.1357 & 96 & 438.1774 \\ 17 & 48.1646 & 37 & 133.6040 & 57 & 230.4539 & 77 & 334.4730 & 97 & 443.7470 \\ 18 & 52.0267 & 38 & 138.2283 & 58 & 235.5057 & 78 & 339.8233 & 98 & 449.3268 \\ 19 & 55.9443 & 39 & 142.8789 & 59 & 240.5747 & 79 & 345.1864 & 99 & 454.9169 \\ 20 & 59.9146 & 40 & 147.5552 & 60 & 245.6607 & 80 & 350.5621 & 100 & 460.5170\end{array}$




$\begin{array}{llllllllll}101 & 466.1272 & 121 & 580.2907 & 141 & 697.7751 & 161 & 818.1061 & 181 & 940.9280 \\ 102 & 471.7472 & 122 & 586.0906 & 142 & 703.7274 & 162 & 824.1906 & 182 & 947.1292 \\ 103 & 477.3771 & 123 & 591.8987 & 143 & 709.6868 & 163 & 830.2813 & 183 & 953.3360 \\ 104 & 483.0167 & 124 & 597.7149 & 144 & 715.6531 & 164 & 836.3781 & 184 & 959.5482 \\ 105 & 488.6658 & 125 & 603.5392 & 145 & 721.6264 & 165 & 842.4810 & 185 & 965.7658 \\ 106 & 494.3245 & 126 & 609.3715 & 146 & 727.6066 & 166 & 848.5900 & 186 & 971.9889 \\ 107 & 499.9927 & 127 & 615.2118 & 147 & 733.5936 & 167 & 854.7050 & 187 & 978.2173 \\ 108 & 505.6702 & 128 & 621.0599 & 148 & 739.5874 & 168 & 860.8260 & 188 & 984.4511 \\ 109 & 511.3569 & 129 & 626.9158 & 149 & 745.5880 & 169 & 866.9529 & 189 & 990.6902 \\ 110 & 517.0528 & 130 & 632.7795 & 150 & 751.5953 & 170 & 873.0857 & 190 & 996.9346 \\ 111 & 522.7579 & 131 & 638.6508 & 151 & 757.6093 & 171 & 879.2245 & 191 & 1003.1842 \\ 112 & 528.4719 & 132 & 644.5299 & 152 & 763.6298 & 172 & 885.3690 & 192 & 1009.4391 \\ 113 & 534.1948 & 133 & 650.4164 & 153 & 769.6570 & 173 & 891.5194 & 193 & 1015.6992 \\ 114 & 539.9266 & 134 & 656.3105 & 154 & 775.6907 & 174 & 897.6756 & 194 & 1021.9645 \\ 115 & 545.6672 & 135 & 662.2121 & 155 & 781.7309 & 175 & 903.8375 & 195 & 1028.2349 \\ 116 & 551.4165 & 136 & 668.1211 & 156 & 787.7775 & 176 & 910.0052 & 196 & 1034.5105 \\ 117 & 557.1743 & 137 & 674.0374 & 157 & 793.8306 & 177 & 916.1785 & 197 & 1040.7911 \\ 118 & 562.9408 & 138 & 679.9610 & 158 & 799.8900 & 178 & 922.3575 & 198 & 1047.0769 \\ 119 & 568.7157 & 139 & 685.8919 & 159 & 805.9558 & 179 & 928.5421 & 199 & 1053.3677 \\ 120 & 574.4990 & 140 & 691.8299 & 160 & 812.0278 & 180 & 934.7322 & 200 & 1059.6635\end{array}$




\section{RÉSUMÉ}

En expérimentation forestière - et dans bien d'autres domaines de la recherche - il n'est pas toujours possible de recueillir les résultats de l'expérimentation sous forme de données quantitatives. Dans ce cas, l'interprétation des résultats expérimentaux par des méthodes qui font appel à l'analyse de variance devient délicate, parfois même impossible en dépit d'artifices classiques tels que la transformation des variables. Pour combler cette lacune, il existe différentes méthodes théoriquement éprouvées mais plus ou moins méconnues des chercheurs qui n'y font que rarement appel. Cet article se propose d'attirer l'attention sur les nombreuses possibilités offertes par l'analyse de l'information - plus spécialement de l'estimation de l'information minima de discrimination - en se limitant à ses applications at cas de la distribution multinomiale, $y$ compris les tables de contingence.

Un certain nombre de résultats mathématiques sont rappelés dans la première partie consacrée à un aperçu théorique sur l'information, l'information minima de discrimination et son estimation. L'intérêt de celle-ci réside dans sa convergence asymptotique à la loi de $\chi^{2}$ dans l'hypothèse nulle.

La seconde partie est consacrée à la comparaison d'échantillons de lois multinomiales - tableaux de fréquences observées - et à l'étude de deux ou plusieurs échantillons, analyse progressive, homogénéité de groupes d'échantillons, test d'indépendance ou d'homogénéité sur les tables de contingence. La plupart des tests sont illustrés par des exemples tirés des dossiers de la Station de Biométrie: ces exemples ont pour but de montrer qu'en dépit d'une complexité formelle apparente les calculs nécessités par l'utilisation de ces tests sont extrêmement simplez et peuvent être réalisés sur la plus simple des machines de bureau dès lors qu'on dispose d'une table numérique de la fonction $\mathrm{n} \log \mathrm{n}$. Une telle table (à 4 décimales) calculée sur l'ordinateur de l'Université de Nancy pour $\mathrm{n} \leqslant 200$ figure en annexe et devrait, dans nombre de cas, s'avérer suffisante.

Comme la somme des carrés dans l'analyse de variance, l'estimation de l'information est susceptible d'ètre décomposée en éléments additifs qui correspondent à différentes sous-hypothèses dont 
l'intersection constitue l'hypothèse globale, Comme telle, elle est intellectuellement très séduisante et il a paru utile de la faire connaitre davantage des chercheurs dans la mesure où elle permet de combler les lacunes d'autres méthodes, parfois utilisées avec une confiance excessive, La théorie de l'information est par ailleurs applicable à bien d'autres lois que la loi multinomiale, notamment à la loi multinormale où elle a donné lieu à d'importants développements. 


\section{SUMMARY}

In forest research - and in many other research fields - it is not always possible to collect the experiment results in the form of quantitative data. For that ground it is difficult, sonetimes impossible, in spite of usual expedients such as the transformation of variables, to interpret the experiment results by means of methods which involve analysis of variance. In order to supply this deficiency several methods have been theoretically worked out but these are more or less known to the research workers who wae them very seldom. In this paper, we intend to draw attention to the numerous possibilities offered by the analysis of information statistics - especially the minimum discrimination information limiting the field of its application to the case of multinomial distribution.

The first part deals with the theoretical aspects of information. the minimum discrimination information and its estimate, and presents some mathematical results. The asymptotic convergence to the $\chi^{2}$ distribution in the mull hypothesis is a most interesting property of the later statistic.

In the second part are compared samples of multinomial distributions - tables of ohserved frequencies - it deals further with the comparison of two or several samples, the successive partition of a set of samples, the homogeneity tests hetween sets of samples. the independence or homogeneity tests for contingency tables. Most tests are exemplified by datas taken out of documents of the Biometry Station: these examples are intended to show that. in spite of an apparent formal complexity, the calculations needed to apply the tests are most simple. and can be carried out with the most common desk machines, since numerical tables of the $n \log n$ function are available. Such a table (with 4 decimals). calculated with the computer of the University of Nancy for $n \leqslant 200$, is given in an appendix, and in many cases should be sufficient.

As also the sum of squares in variance analysis, the information statistic can be split into additive elements, which correspond to different sub-hypothesis, the intersection of which expresses the whole hypothesis. Just as it is, it is intellectually attractive and it has been thought useful to make it known to research workers in 
so far as it enables to supply the deficiencies of other methods, sometimes considered as most reliable ones. The information theory is besides suitable to many others distributions than the multinomial especially to the multinormal, where it led to important developments. 


\section{ZUSAMMENFASSUNG}

In der forstlichen Forschung und auch auf vielen anderen Forschungsgebieten ist es nicht inmer möglich die Versuchsergebnisse in der Form quantitativer Daten zu sammeln. In diesem Falle wird die Interpretation von Versuchsergebnissen, die mit Methoden der Varianzanalyse gewonnen werden delikat, manchmal aber auch unmöglich, trotz Anwendung klassischer Kunstgriffe wie zum Beispiel die Transformation der Variablen. Un diese Lücke zu schliessen gibt es verschiedene theoretisch erprobte Methoden, die den Forschern jedoch meist mehr oder weniger bekannt sind und daher selten angewandt werden. Die vorliegende Arbeit möchte die Aufmerksamkeit auf die zahlreichen Möglichkeiten der Informationsanalyse lenken — insbesondere auf die Schätzung der Minimalinformation der Trennung wohei jedoch die Anwendung auf Fälle der multinomialen Verteilung - die Kontingenztafeln inbegriffen - beschränkt wird.

Im ersten Teil werden eine gewisse Anzahl von mathematischen Ergebnissen in Erinnerung gerufen und eine kurze theoretische Darstellung erläutert die Begriffe Information, Minimalinformation der Trennung und seine Schätzung. Die Bedeutung der Minimalinformation der Trennung liegt in ihrer asymptotischen Konvergenz mit der $\chi^{2}$ Verteilung bei Zugrundelegung der Nullhypothese.

Der zweite Teil behandelt den Vergleich won Stichproben multinomialer Verteilungen, - Tabellen der beobachteten Hãufigkeiten - die Untersuchung zweier oder mehrerer Stichproben, die progressive Analyse, die Homogenität von Gruppen von Stichprohen, sowie die Prüfung der Unabhängigkeit oder der Homogenität von Kontingenztafeln. Die meisten dieser Teste werden an Beispeilen aus den Archiven der Station für Biometrie erläutert. Diese Beispiele sollen zeigen, dass trotz der offensichtlich komplexen Form, die zur Anwendung dieser Teste notwendigen Berechnungen äusserst einfach sind und mit jeder Bürorechenmaschine durchgeführt werden können, wenn eine numerische Tafel der Funktion $n \log n$ zur Verfügung steht. Eine solche Tafel mit 4 Dezimalen wurde auf einem Elektronenrechner der Universität Nancy für $n \leqslant 200$ berechnet und in Anhang beigegeben. Die Tafel dürfte sich in vielen Fällen als ausreichend erweisen. 
Ebenso wie die Summe der Quadrate bei der Varianzanalyse, eignet sich auch der Schätzwert der Information für eine Zerlegung in additive Elemente die bestimmten Subhypothesen entsprechen und deren Intersektion die Globalhypothese darstellt. Als solcher ist der Schätzwert der Information, intellektuell gesehen als sehr verlockend zu betrachten und es erschien zweckmässig, die Forscher näher damit bekannt zu machen. Dies gilt vor allem für jene Fälle, wo der Schätzwert der Information es ermöglicht andere Methoden zu vervollständigen, die oftmals mit einem zu grossen Vertrauen angewendet werden. Die Theorie der Information ist im Ubrigen neben der multinomialen Verteilung auch auf eine Anzahl anderer Verteilungen anwendbar, vor allem auf die multinormale Verteilung, wo sie eine bedeutende Entwicklung ermöglichte. 